\title{
When the Milky Way turned off the lights: APOGEE provides evidence of star formation quenching in our Galaxy
}

\author{
M. Haywood ${ }^{1}$, M. D. Lehnert ${ }^{2}$, P. Di Matteo ${ }^{1}$, O. Snaith ${ }^{3}$, M. Schultheis ${ }^{4}$, D. Katz ${ }^{1}$, and A. Gómez ${ }^{1}$ \\ ${ }^{1}$ GEPI, Observatoire de Paris, CNRS, Université Paris Diderot, 5 place Jules Janssen, 92190 Meudon, France \\ e-mail: Misha.Haywood@obspm.fr \\ 2 Institut d'Astrophysique de Paris, CNRS UMR 7095, Université Pierre et Marie Curie, 98bis Bd Arago, 75014 Paris, France \\ 3 School of Physics, Korea Institute for Advanced Study, 85 Hoegiro, Dongdaemun-gu, Seoul 02455, Republic of Korea \\ ${ }^{4}$ Université de Nice Sophia-Antipolis, CNRS, Observatoire de Côte d'Azur, Laboratoire Lagrange, 06304 Nice Cedex 4, France
}

Received 15 October 2015 / Accepted 5 January 2016

\section{ABSTRACT}

\begin{abstract}
Quenching, the cessation of star formation, is one of the most significant events in the life cycle of galaxies. While quenching is generally thought to be linked to their central regions, the mechanism responsible for it is not known and may not even be unique. We show here the first evidence that the Milky Way experienced a generalised quenching of its star formation at the end of its thick-disk formation $\sim 9 \mathrm{Gyr}$ ago. The fossil record imprinted on the elemental abundances of stars studied in the solar vicinity and as part of the APOGEE survey (APOGEE is part of the Sloan Digital Sky Survey III) reveals indeed that in less than $\sim 2$ Gyr (from 10 to 8 Gyr ago) the star formation rate in our Galaxy dropped by an order of magnitude. Because of the tight correlation that exists between age and $\alpha$ abundance, the general cessation of the star formation activity reflects in the dearth of stars along the inner-disk sequence in the $[\mathrm{Fe} / \mathrm{H}]-[\alpha / \mathrm{Fe}]$ plane. Before this phase, which lasted about $1.5 \mathrm{Gyr}$, the Milky Way was actively forming stars. Afterwards, the star formation resumed at a much lower level to form the thin disk. These events observed in our Galaxy are very well matched by the latest observation of MW-type progenitors at high redshifts. In late-type galaxies, the quenching mechanism is believed to be related to a long and secular exhaustion of gas. Our results show that in the Milky Way, the shut-down occurred on a much shorter timescale, while the chemical continuity between the stellar populations formed before and after the quenching indicates that it is not the exhaustion of the gas that was responsible for the cessation of the star formation. While quenching is generally associated with spheroids in the literature, our results show that it also occurs in galaxies like the Milky Way, where the classical bulge is thought to be small or non-existent, possibly when they are undergoing a morphological transition from thick to thin disks. Given the demographics of late-type galaxies in the local Universe, in which classical bulges are rare, we suggest further that this may hold true generally in galaxies with mass lower than or approximately of $M^{*}$, where quenching could directly be a consequence of thick-disk formation, while quenching may be related to development of spheroids in higher mass galaxies. We emphasize that the quenching phase in the Milky Way could be contemporaneous with, and related to, the formation of the bar, at the end of the thick-disk phase. We sketch a scenario on how a strong bar may inhibit star formation.
\end{abstract}

Key words. Galaxy: abundances - Galaxy: disk - Galaxy: evolution - galaxies: evolution

\section{Introduction}

Galaxies are observed to quench their star formation activity at an epoch that depends on their mass (e.g. Cimatti et al. 2006), with the most massive galaxies quenching at $z \sim 2-3$ (Cimatti et al. 2004). Whether this quenching is a complete shut-down of star formation or a more gradual transition from an active phase of star formation to a more quiescent one is still debated. Quenching in massive galaxies seems to be rapid (e.g. Mancini et al. 2015), and possibly slower in spiral galaxies (Schawinski et al. 2014). Various mechanisms have been proposed to explain why galaxies quench as a function of mass and epoch. The first class of proposed mechanisms either removes the gas from the system or prevent further gas accretion. This is the case for powerful feedback from active galactic nuclei, the socalled AGN feedback (Granato et al. 2004), which could sweep the gas from galaxies, but it is thought to be effective only for the most massive galaxies (see e.g. Förster Schreiber et al. 2014; Gabor \& Bournaud 2014), while feedback from supernovae could be effective for less massive objects (Kaviraj et al. 2007). Gas accretion can be halted by heating circum-galactic gas (e.g. radio-mode AGN feedback; Croton et al. 2006) or prevent accretion of additional cold gas through virial shock heating (Birnboim \& Dekel 2003). The second class of mechanisms inhibits star formation either through stabilization of a disk galaxy as it is transformed to a spheroid (also known as morphological quenching; Martig et al. 2009) or dust heating by radiation of low-mass stars (Kajisawa et al. 2015; Conroy et al. 2015), or high levels of turbulence generated by an AGN (Guillard et al. 2015).

Recent results also suggest that quenching in disks is related to the mass build-up of bulges and morphological change in galaxies, perhaps marking the end of the bulge building phase (Bluck et al. 2014; Lang et al. 2014; Abramson et al. 2014; Bell et al. 2012). For Milky Way-type galaxies, selected to have comoving number densities similar to that of galaxies with stellar mass similar to the Milky Way (hereafter MW) at $z \sim 0$, quenching seems to occur at $z \sim 1.6$ (Morishita et al. 2015) in the inner parts of disks $(<2.5 \mathrm{kpc})$. Because most of the star formation since $z \sim 2-3$ occurred in Milky Way-like disk galaxies (e.g. Nelson et al. 2013), which are the dominant type on the main sequence, understanding if and how star formation quenching 
occurred in our Galaxy is of paramount importance. We argue in the present paper that the MW provides the nearest example of quenching in a galaxy, occurring 9-10 Gyr ago at the transition from the thick to the thin disks, in agreement with what is measured on high-redshift galaxies.

While several papers have suggested that the thick disk could be formed in a burst phase of star formation more than $10 \mathrm{Gyr}$ ago (e.g. Burkert et al. 1992; Chiappini et al. 1999; Fuhrmann 2004; Reid 2005; Bernkopf \& Fuhrmann 2006), we argued in Haywood et al. (2013) that the thick-disk population extended from about 13 to $9 \mathrm{Gyr}$ ago. Indeed, the modelling of solar vicinity chemical abundances in Snaith et al. $(2014,2015)$ has shown that the inner $(<10 \mathrm{kpc})$ disk $^{1}$ of our Galaxy shows two distinct phases of star formation, see Fig. 1. The first corresponds to the formation of the thick disk between 13 and $9 \mathrm{Gyr}$, the second phase between $7 \mathrm{Gyr}$ and the present epoch corresponds to the formation of the thin disk. At the end of the thick-disk phase, between 10 and $9 \mathrm{Gyr}$, the star formation in the MW dropped from a high-intensity starburst regime (see also Lehnert et al. 2014) to a more quiescent phase at $z<1$. At this epoch, the decrease in the star formation rate (SFR) was much faster than expected from the exhaustion of gas as provided by a SchmidtKennicutt relation. This implies that star formation ceased not due to a shortage of gas, but because star formation was inhibited and particularly inefficient (i.e. had very long gas depletion times). Between $\sim 8.5$ and $7.5 \mathrm{Gyr}$, the star formation history (SFH) of the MW shows a prominent dip, compatible with very low rates of, or no star formation during this period. Although galaxies are expected to experience fluctuations in their SFR, this dip is made peculiar by marking the end of the growth of the thick disk and the transition to another dynamical and morphological stellar population. In the present study, we argue that this cessation of the SFR corresponds to star formation quenching that is observed in galaxies at high redshifts. Using studies of the demographics of disks with mass lower than $M^{*}$, the characteristic stellar mass of the galaxy stellar mass function, in the local volume, we argue also that quenching seen in Milky Waylike disks galaxies and later types at high redshifts is not bulge building (e.g. Abramson et al. 2014), but represents the end of thick-disk building.

Snaith et al. (2015) found the dip in the SFH by fitting a model to the age- $[\alpha / \mathrm{Fe}]$ relation of solar vicinity stars. Because the dip occurs at the end of the thick-disk phase, an epoch when stars tend to form on more circular orbits, it was not clear whether this feature was only localized around the solar circle or if it was more pervasive, happening throughout the galactic disk. Orbits becoming more circular means that stars from the inner disk may not be fairly represented at the solar circle, and thus any features in the SFH are only constrained for the evolution of the MW at around the radius at which the Sun lies from the Galactic centre. It is important at this stage to emphasize that the SFH was derived from fitting the age- $[\mathrm{Si} / \mathrm{Fe}]$ relation obtained from an inhomogeneous and incomplete sample. However, since we did not rely on stellar densities, this has no or limited impact

\footnotetext{
We use here the nomenclature introduced in Haywood et al. (2013, 2015; Snaith et al. 2015), The inner disk is made of stars that belong to both the thick and thin disks and form the sequence that extends from metal-poor $\alpha$-rich abundances to the metal-rich $\alpha$-poor sequence. It dominates the chemical patterns, as seen in the APOGEE data at $R<$ $7 \mathrm{kpc}$, see Fig. 3. This sequence is outlined by our model in both Fig. 2 for solar vicinity data and Fig. 3 for APOGEE data. The outer disk is defined by the low- $\alpha$ sequence, visible in the solar vicinity chemical patterns (see Haywood 2008), and now beautifully illustrated by the APOGEE survey with in situ stars.
}

because as far as the abundance patterns observed in Adibekyan et al. are correct and representative, the age- $[\mathrm{Si} / \mathrm{Fe}]$ will be a faithful record of the past star formation rate. We also note that Nissen (2015) confirmed the existence of a tight correlation between $\alpha$ elements and age in the thin-disk regime. Indeed, that the abundance patterns of the Adibekyan et al. sample are correct and representative can be checked by comparing with similar samples (e.g. Bensby et al. 2014) or, more extensively, on the APOGEE data (Majewski et al. 2015), Fig. 3. Therefore, the SFH derived in Snaith et al. (2015) is valid for the whole inner disk, as far as the stars that enter the age- $[\mathrm{Si} / \mathrm{Fe}]$ relation are representative of the whole disk. Derivation of the orbital properties of these stars in Haywood et al. (2015), Fig. 4, which shows the pericenters of stars as a function of their $\alpha$ abundance and age, illustrates that this must be the case for stars older than about 8-9 Gyr (thick-disk stars), which reach the innermost regions of the disk, but it must be checked for younger stars.

The aim of the present paper is therefore to confirm the validity over the whole disk of a SFH measured locally, and in particular that of the dip seen between 9 and 7 Gyr, by comparing our model with star counts several kpc from the Sun. We demonstrate that this dip is visible throughout the inner disk $(R<10 \mathrm{kpc})-$ it is a general feature in the global evolution of the SFH of the MW.

The outline of this paper is as follows: in the next section, we briefly describe our model and discuss the evidence that the star formation in our Galaxy ceased about 8 Gyr ago from spectroscopic and age data of a sample of stars in the solar vicinity. In Sect. 3 we present our selection of the APOGEE data and the comparisons with our model. We discuss these results in Sect. 4 and in particular its significance for the evolution of Milky Waytype galaxies generally. We provide what we conclude from this study in Sect. 5.

\section{Model and evidence from the solar vicinity}

The interpretation of the solar vicinity chemical patterns is paved with a rich literature (Matteucci \& Francois 1989; Gilmore \& Wyse 1991; Timmes et al. 1995; Chiappini et al. 1997; Haywood 2001; Schönrich \& Binney 2009; Kobayashi et al. 2011). Haywood et al. (2013) emphasized that the low- $\alpha$ and $\alpha$-rich sequences in the $[\alpha / \mathrm{Fe}]-[\mathrm{Fe} / \mathrm{H}]$ plane (see Fig. 2 ) are possibly the result of two different chemical evolutionary histories, those of the inner and outer disks. We can sample these two sequences at the solar vicinity because the Sun is at the interface between them. These two chemical evolutions, having widely different metallicities at similar ages, cannot be represented by a single model (Haywood et al. 2013). We emphasized that while the inner-disk sequence is typical of a closed-box evolution, the lower- $\alpha$ sequence is representative of chemical evolution with dilution. These models were described and compared to chemical trends in the solar vicinity in Snaith et al. (2015). Hallé et al. (2015) proposed that the outer Lindblad resonance (OLR) of the bar, located in the solar vicinity (Dehnen 2000), is responsible for keeping these two parts of the disks relatively well separated. APOGEE data have confirmed these results by showing that the inner disk essentially lacks low- $\alpha$ stars, while the outer disk is dominated by the low- $\alpha$ sequence (e.g. Hayden et al. 2015). The SFH we derived in Snaith et al. (2015) is the general SFH of the inner disk $(R<10 \mathrm{kpc})$, and the quenching event described here applies to the inner disk only, not the outer disk. As shown in Snaith et al. (2015), the outer disk (beyond the OLR) is characterized by different chemical patterns that translate into a different, flatter, SFH. Hereafter we analyse the inner-disk sequence 
only, which extends from the $\alpha$-rich metal-poor to the $\alpha$-poor metal-rich stars. Although this sequence is often called the thickdisk sequence, we prefer to call it the inner-disk sequence because we think it describes the evolution of the whole inner disk, comprising both the thick and thin disks (essentially pure inner disk at $R<7 \mathrm{kpc}$, and a mix of it with the outer disk, between 7 and $10 \mathrm{kpc}$, the OLR region).

\subsection{Chemical evolution model and derived SFH}

The level of $[\alpha / \mathrm{Fe}]$ abundances at a given time is determined by the ratio of the present to past SFR (Gilmore \& Wyse 1991). The higher the past SFR (relative to the present SFR), the lower the $[\alpha / \mathrm{Fe}]$ abundance at more recent times, implying a steep decrease in $[\alpha / \mathrm{Fe}]$ abundance, and a steeper slope in the age$[\alpha / \mathrm{Fe}]$ abundance relation. The slope of this relation reflects past SF intensity. This is illustrated by the SFHs of Fig. $4 \mathrm{a}$ and the corresponding age- $[\alpha / \mathrm{Fe}]$ relations of Fig. 5. The information on the SFH of the disk is therefore fossilized in the run of $\alpha$-element abundances with stellar age in the solar vicinity (Haywood et al. 2013) and has been used in Snaith et al. (2014, 2015) to derive a general history of the star formation in the MW by fitting a chemical track to the age- $[\mathrm{Si} / \mathrm{Fe}]$ relation. To fit the age- $[\alpha / \mathrm{Fe}]$ relation, a chemical evolution model must be assumed. We adopted a closed-box model for the reasons given in Haywood (2014a,b) and Snaith et al. (2015). We emphasized that the closed-box is taken here to mean a model where most accretion has occurred early (before substantial star formation occurred) in the inner disk, motivated by expectations that infall in the central regions of galaxies must be rapid (Haywood et al. 2015). Snaith et al. $(2014,2015)$ did not assume any dependency of the SFR on the gas density, as is typically done in chemical evolution models, but derived the SFH that allows the model to best fit the age-[Si/Fe] relation (see also Fig. 1). Detailed accounts of the procedure used to fit the stellar age- $[\mathrm{Si} / \mathrm{Fe}]$ relation, its robustness and uncertainties of the method and result were given in these two papers. Detailed comparisons between our model and the observed MDF from APOGEE are the focus of a forthcoming article.

Figure 1 also shows the evolution of the gas fraction as a function of time by our model. It shows that at the end of the thick disk phase $(9.5 \mathrm{Gyr}, z=1.5)$, the amount of gas in the system is still very large $(\sim 50 \%)$. Similar fractions have been reported on massive galaxies at these redshifts, see for instance Daddi et al. (2010) or Béthermin et al. (2015), but it remains to be confirmed on MW-type galaxies ${ }^{2}$.

The model is able to follow the evolution of silicon, magnesium, and oxygen. Because of problems with magnesium theoretical yields, however, it is difficult to compute a mean $\alpha$ abundance from these elements to compare to the APOGEE data. Silicon theoretical yields are well behaved and have been used to constrain our model. However, the silicon abundances in the APOGEE data are significantly different from the silicon abundance determined for local samples of stars. To avoid being trapped in the details of the behaviour of one particular element, we decided to compare mean $\alpha$ abundances. To do so, we converted the silicon model abundance to $[\alpha / \mathrm{Fe}]$ using a relation

\footnotetext{
2 This is also compatible with the gas fractions that are implied by the derived SFHs for MW-type galaxies. For instance, Papovich et al. (2015) inferred that while the gas fraction in M31-type galaxies has decreased to lower than $20 \%$ at $z \sim 1.5$, it is still high for MW-sized galaxies at $\sim 40 \%$, consistent with the fraction estimated here. See also Popping et al. (2015).
}

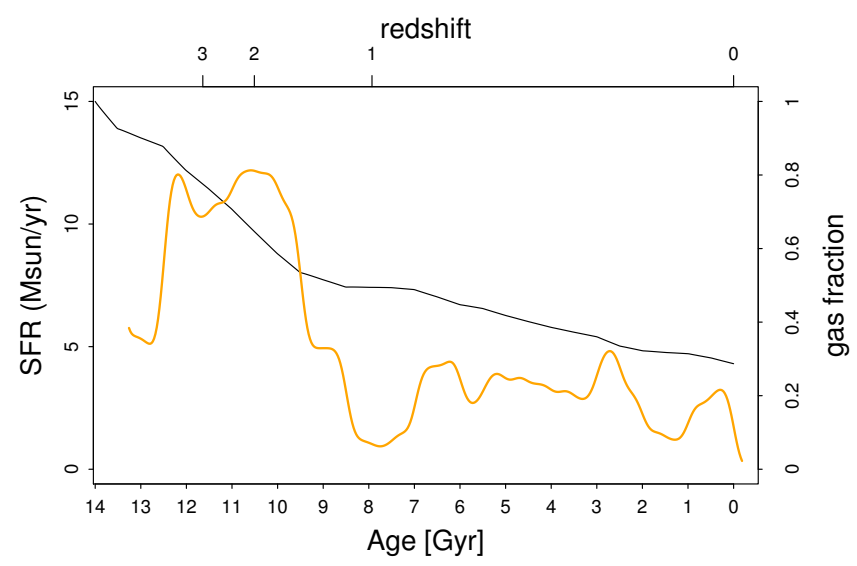

Fig. 1. Star formation history of the inner Milky Way $(R<10 \mathrm{kpc}) \mathrm{de}-$ rived from fitting the solar vicinity age- $[\mathrm{Si} / \mathrm{Fe}]$ abundance with a chemical evolution model in Snaith et al. (2014; 2015; orange curve, left axis), together with the gas fraction evolution in the model (thin black curve and right axis). The SFR at $\sim 8$ Gyr is negligible (consistent with no star formation), while the gas fraction is still very high in the system, similar to the molecular gas fractions estimated in disks at redshifts 1-3 (Daddi et al. 2010; Arevana et al. 2010; Dannerbauer et al. 2009; Tacconi et al. 2010, 2013). The SFH is normalised such that the current stellar mass of the Milky Way is $5 \times 10^{10} M_{\odot}$.

obtained from the observed $[\mathrm{Si} / \mathrm{Fe}]$ and $[\alpha / \mathrm{Fe}]$ of the Adibekyan et al. (2012) sample. The model was then compared to the data (Fig. 2). This is justified by the surprisingly close similarity between the behaviour of the $\alpha$ solar vicinity abundance scales and from APOGEE, as corroborated by the distributions of Fig. 3, although they are derived from different data and elements. The comparison of the $[\alpha / \mathrm{Fe}]$ distributions between the two samples shows that the systematic shift between the two is at most $0.02-0.03$ dex and is thus insignificant for our analysis.

\subsection{What do we wish to measure?}

Figure 1 shows that the SFR of the MW thick and thin disks at $R<10 \mathrm{kpc}$ has dropped suddenly $10 \mathrm{Gyr}$ ago to reach a minimum $\sim 8$ Gyr ago. As mentioned previously, this event has been found in Snaith et al. (2014) by fitting the age-[Si/Fe] relation. We now wish to search for its signature in the star counts. Because of the tight correlation that exists between age and $[\alpha / \mathrm{Fe}]$ (Haywood et al. 2013, 2015), we expect any variation in the SFH to be imprinted on the number density of stars as a function of $[\alpha / \mathrm{Fe}]$ and to a minimum in the SFH should correspond a minimum, a dip, or a bimodality in the stellar counts as a function of $[\alpha / \mathrm{Fe}]$. Now, we must specify where we wish to measure the variation of densities in $[\alpha / \mathrm{Fe}]$. When slicing the $[\mathrm{Fe} / \mathrm{H}]-[\alpha / \mathrm{Fe}]$ plane at constant $[\mathrm{Fe} / \mathrm{H}]$ (i.e., vertically), a bimodality arises because we successively cross the relatively $\alpha$-rich and then $\alpha$-poor sequences. The chemical evolution from the thick to the thin disk has been described as parallel tracks connecting these two sequences (e.g. Schönrich Binney 2009, their Figs. 3 and 4). In our view, however, for reasons given in the previous section, the chemical evolution does not connect the two sequences. The bimodality that arises from the dip that separates the $\alpha$-rich sequence from the metal-poor thin-disk sequence when slicing the $[\mathrm{Fe} / \mathrm{H}]-[\alpha / \mathrm{Fe}]$ plane vertically has no evolutionary meaning (see Nidever et al. 2014; or Kordopatis et al. 2015, for a characterization of the distance that separates the two sequences). 


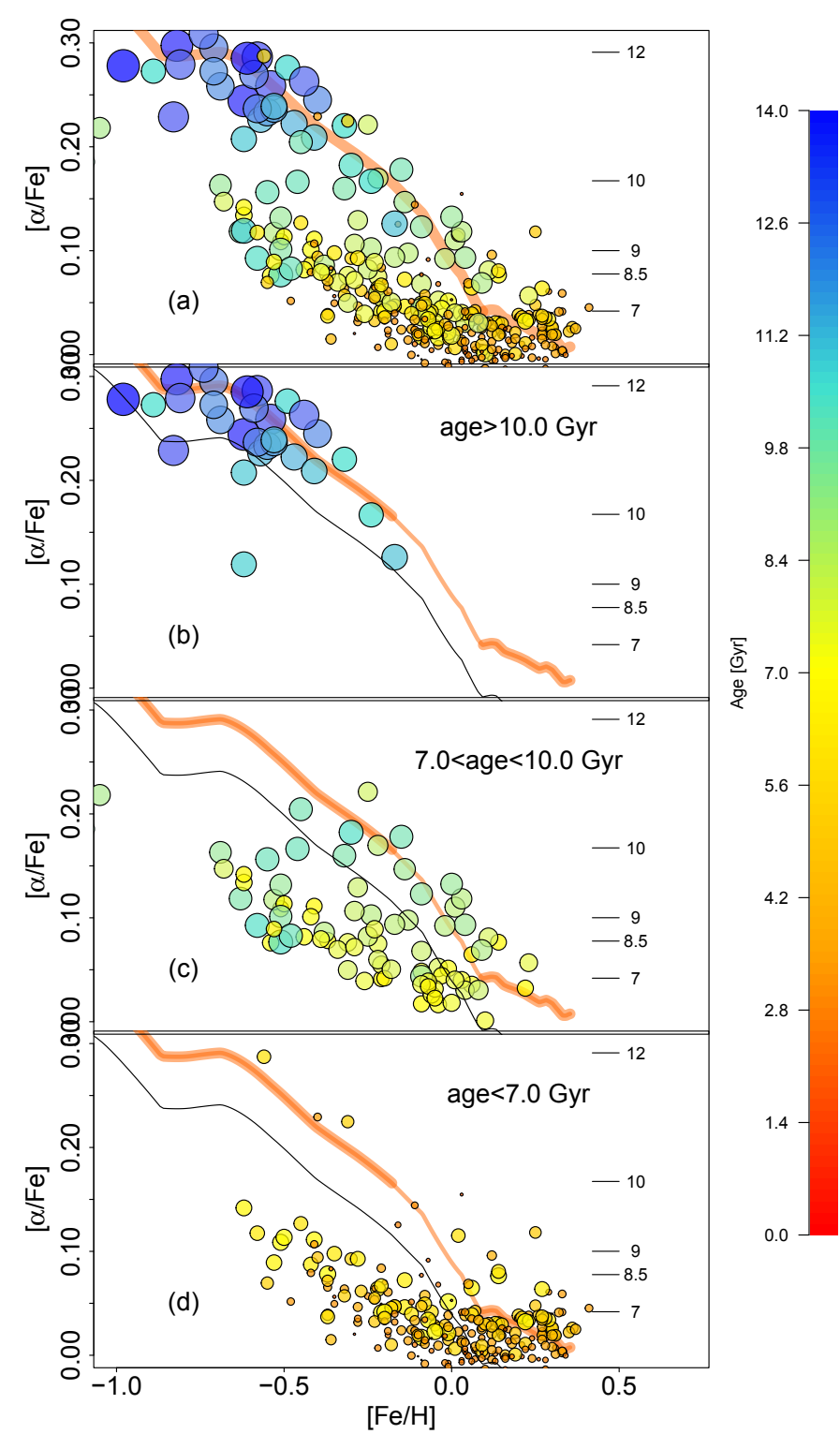

Fig. 2. $[\alpha / \mathrm{Fe}]-[\mathrm{Fe} / \mathrm{H}]$ distribution at the solar vicinity for the sample of Adibekyan et al. (2012) with ages from Haywood et al. (2013). The colours (indicated in the legend) and size of the symbols indicate the age of the stars (larger circles imply older ages). The orange line represents the model of Snaith et al. (2015), which has the SFH of Fig. 1. The thinner segment of the line corresponds to the lull in the SFH of Fig. 1, between 10 and $7 \mathrm{Gyr}$ ago. The thin black curve is the same model shifted down by -0.05 dex and is used in Sect. 3 to select inner-disk stars from the APOGEE survey. The stars below and to the left of this line are either accreted stars according to Nissen \& Schuster (2010; for those objects which have $[\mathrm{Fe} / \mathrm{H}]<-0.7 \mathrm{dex}$ ), or metal-poor thin-disk stars. A vertical scale within each plot indicates ages along the model from 7 to $12 \mathrm{Gyr}$.

The evolution of the inner disk occurs along a single welldefined sequence in the $[\mathrm{Fe} / \mathrm{H}]-[\alpha / \mathrm{Fe}]$ plane, which reaches from metal-poor $\alpha$-rich $([\alpha / \mathrm{Fe}] \sim 0.3 \mathrm{dex})$ stars to $\alpha$-poor metal-rich objects $([\alpha / \mathrm{Fe}] \sim 0$. dex, $[\mathrm{Fe} / \mathrm{H}]>0.0 \mathrm{dex})$. This sequence is well defined observationally in the data of Adibekyan et al. (2012), see our Fig. 2, and well reproduced by our model (orange curve). The bimodality in which we are interested in here is measured along this sequence and not at constant $[\mathrm{Fe} / \mathrm{H}]$.
Figure 2 shows the $[\mathrm{Fe} / \mathrm{H}]-[\alpha / \mathrm{Fe}]$ distribution for a sample of $F$ and $G$ dwarfs from Adibekyan et al. (2012) for which ages could be determined (Haywood et al. 2013). The same stars have been used to derive the SFH in Snaith et al. (2014, 2015, and Fig. 1) by fitting the age- $[\mathrm{Si} / \mathrm{Fe}]$ relation. The $[\mathrm{Fe} / \mathrm{H}]-[\alpha / \mathrm{Fe}]$ chemical track corresponding to this SFH has been overplotted on the data. We note that it is not a fit to the data, but results directly from the fit made from the age- $[\mathrm{Si} / \mathrm{Fe}]$ relation. We converted $[\mathrm{Si} / \mathrm{Fe}]$ to $[\alpha / \mathrm{Fe}]$ using a regression fit between these two quantities for stars in the Adibekyan sample. This model corresponds to the inner-disk sequence and is shown as a continuous line from the old $\alpha$-rich stars to the metal-rich $\alpha$ poor objects. We emphasize that this model describes the evolution of the innerdisk sequence alone. It does not represent the lower- $\alpha$ sequence with $[\mathrm{Fe} / \mathrm{H}]<0.0$ dex. The black curve in Fig. 2 is the inner-disk sequence shifted by -0.05 dex. The inner-disk stars were chosen such that they are above this limit. In the following sections, we use this criterion to generally select the inner-disk stars.

The age scales in Fig. 2 - both the observational scale as illustrated by the symbols and the model scale - indicate that the inner-disk sequence is a temporal sequence. Figures 2, panels $b-d$ show the $[\alpha / \mathrm{Fe}]-[\mathrm{Fe} / \mathrm{H}]$ abundance distribution dividing the sample in three different age intervals. The model track is separated into three segments, with the one between 10 and 7 Gyr corresponding to the lull in the SFH of Fig. 1; it is represented by a thinner line. Hence, we expect that a pause between 7 and 10 Gyr in the SFH corresponds to a significantly lower density of stars between about 0.05 and 0.17 dex.

There is indeed a hint of a minimum density in this interval when we plot the histogram of $[\alpha / \mathrm{Fe}]$ for stars in the Adibekyan et al. (2012) sample (see Fig. 3a). Given the nature of the sample used here, however, which is both not complete over its volume and has limited numbers of stars, any dip in the histogram is difficult to discern. Because of these limitations, we now turn to the APOGEE survey with its higher completion and significantly better statistics (Holtzman et al. 2015). Implicit in the present work is the assumption that the age- $[\alpha / \mathrm{Fe}]$ relation deduced from stars in the solar vicinity is valid over the inner (thin and thick) disk. This assumption is based on our previous study (Haywood et al. 2015), where we have shown that the tight correlation observed in the solar vicinity between age and $\alpha$ abundances strongly favours a uniform SFH over the entire radial distribution of the inner disk. Haywood et al. (2015, see their Fig. 4) showed that the pericenters of the thick-disk stars in the sample reach the innermost Galaxy (pericenters $<2 \mathrm{kpc}$ ). Hence, the sample, although local, probes the entire inner disk.

As mentioned at the beginning of this section, the speed at which $[\alpha / \mathrm{Fe}]$ abundance varies with time is a function of the star formation intensity. Because of the wide orbital range of the stars sampled in the solar vicinity, the observed tight correlation between age and $[\alpha / \mathrm{Fe}]$ abundance supports a homogeneous SFH in the inner disk.

\section{Archeology of a quenching event in the Milky Way star formation history: stellar densities}

\subsection{Selected data}

Starting from a sample of 83168 stars with distances, our selection of APOGEE objects is similar to the one made by the APOGEE collaboration (Hayden et al. 2015): we chose objects with $T_{\text {eff }}<5500 \mathrm{~K}$ and $1.0<\log g<3.8$. Since the determination of $[\alpha / \mathrm{M}]$ may be less reliable (Holtzman et al. 2015) below 


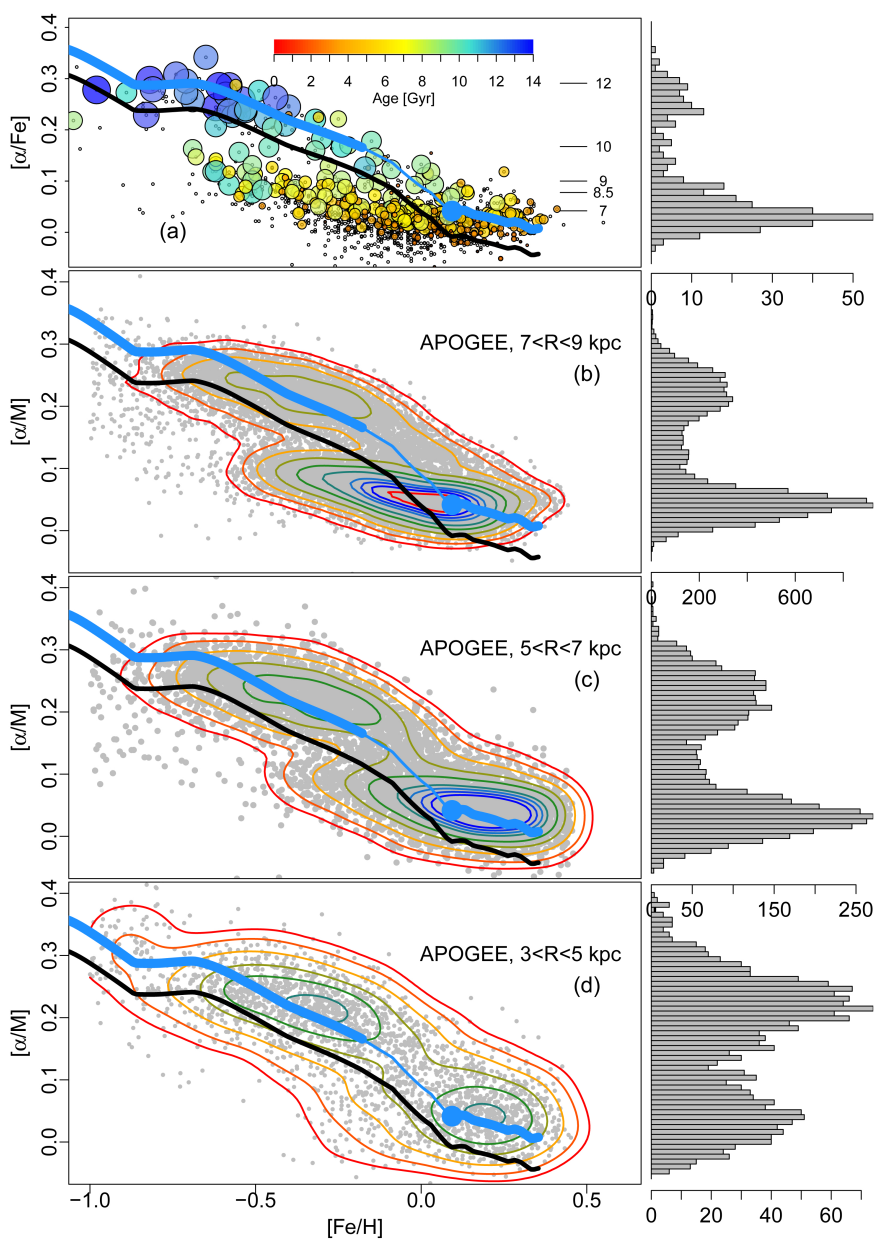

Fig. 3. a) Abundance distributions for stars in the solar vicinity from very high resolution spectroscopic data and $\mathbf{b}$ )-d) data from APOGEE. The inner-disk sequence (above the black line) is composed of thickdisk and metal-rich thin-disk stars and dominates the inner-disk stellar populations, as illustrated in c) and d). The blue thick curve represents the track of our chemical evolution model, with the dot indicating the beginning of the thin-disk era at $7 \mathrm{Gyr}$. The thinner blue segment indicates the quenching phase from 10 to 7 Gyr. The black line is defined as the model -0.05 dex in $[\alpha / \mathrm{Fe}]$. We emphasize that the model (blue line) represents the evolution of the inner-disk sequence alone (stars above the black line). The outer-disk sequence (below the black line) represents a different evolution and requires a different model (see Snaith et al. 2015). The histograms count the number of stars above the black line. In a), grey points are all the stars in the solar vicinity sample, while coloured circles are a subsample for stars with age. The size and colour of the symbols code the age of the stars. The tick marks indicate the age of the model along the track. The histogram includes all stars above the black line (whether they have an age determination or not).

$4000 \mathrm{~K}$, we also removed objects below this limit, amounting to 35965 stars between 3 and $9 \mathrm{kpc}$ from the Galactic centre.

Distances derived for the whole APOGEE survey were taken from Schultheis et al. (2014). They have been derived using the Padova set of isochrones. For each star, the closest point on the isochrones was searched for in the $[\mathrm{M} / \mathrm{H}], \log g, T_{\text {eff }}$ space. Stars for which a point on the grid closer than $500 \mathrm{~K}$ in temperature or $0.5 \mathrm{dex}$ in $\log g$ could not be found were discarded. In addition, $3 \%$ of the stars do not fall within the grid of isochrones and were also discarded. Absolute magnitudes in the 2MASS photometric bands were estimated from this procedure and were used to estimate both the extinction and the distance of each
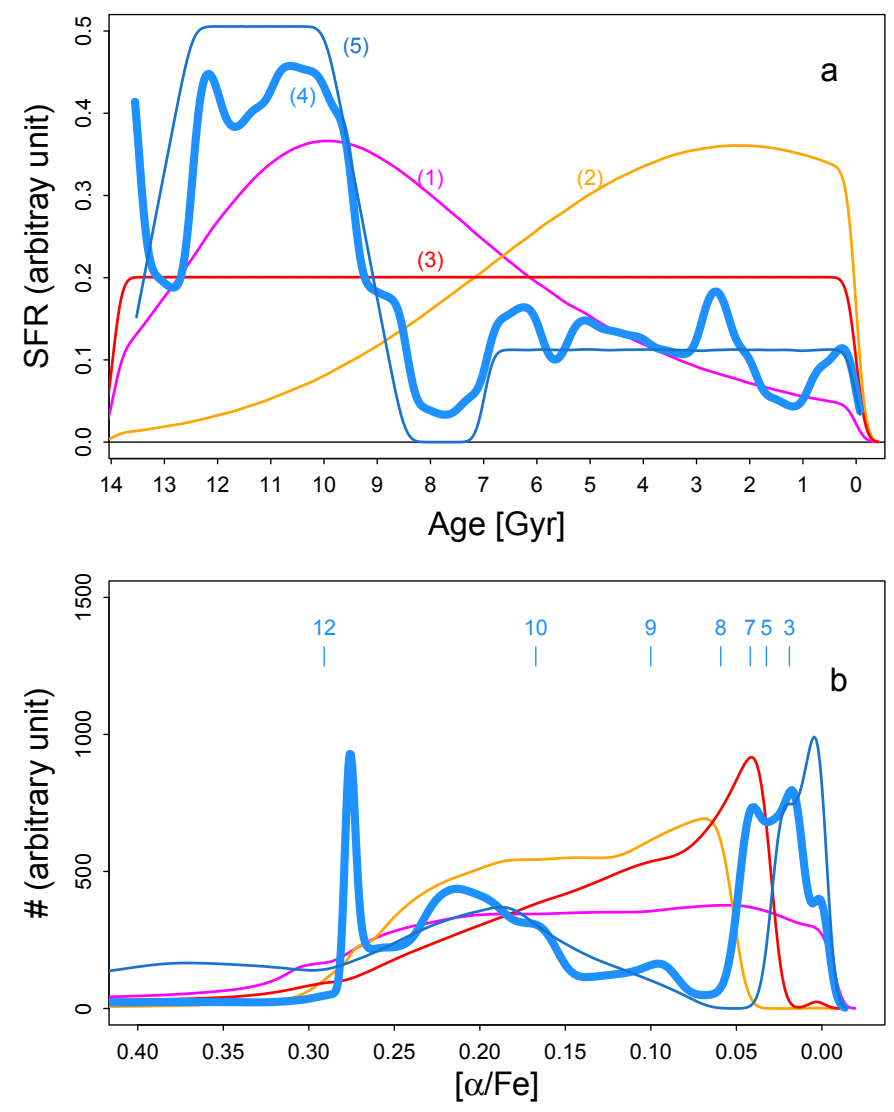

Fig. 4. a) Broad range of SFHs designed to cover decreasing, increasing, and constant star-formation rates with time (labelled 1,2,3), and the SFH from Snaith et al. (2014, 2015), with a dip between 8.5 and 7 Gyr (thick blue curve labelled 4). SFH 5 is similar to 4 with noise removed (thin dark blue curve). b) The corresponding stellar densities as a function of $[\alpha / \mathrm{Fe}]$. SFHs like 1,2 , and 3 do not generate a dip in the $[\alpha / \mathrm{Fe}]$ distribution. Only an SFH like 4 (or 5) with a drop and cessation in star formation in the specific time interval between $\sim 10$ to $7 \mathrm{Gyr}$ can generate a distribution with a significant dip (blue curve). The short blue vertical lines indicate the time variation in Gyr for model 4 (thick blue curve). The peak at $[\alpha / \mathrm{Fe}] \sim 0.28 \mathrm{dex}$ is due to the flattening of the variation of $[\alpha / \mathrm{Fe}]$ visible in Fig. 1, which is not at all significant compared to the data used to generate the SFH (Snaith et al. 2014, 2015).

star. The median error in distance estimates, taking into account the uncertainties on the atmospheric parameters from APOGEE, is of about $30-40 \%$ (Schultheis et al. 2014) $)^{3}$. Relying on red clump stars only would have provided more accurate distances. However, the APOGEE-RC catalogue (see Bovy et al. 2014), containing 10341 objects, with the majority at Galactocentric distances larger than $8 \mathrm{kpc}$, does not sample the inner disk particularly well.

We now apply the same selection as for the sample of solar vicinity stars, see Fig. 3. The blue curve represents the model of the inner galactic disk described in the previous section. The black curve is the same model lowered by $0.05 \mathrm{dex}$, and we select stars with a good probability of belonging to the inner-disk sequence as those stars above this limit. This limit is somewhat arbitrary, but it was chosen to select stars on the $\alpha$-rich, innerdisk sequence and exclude those on the $\alpha$-poor (outer) thin-disk sequence. It must be noted that solar metallicity stars that belong

3 The distances were also compared by one of the authors (M. Schultheis) to Hayden et al. (2014) and Santiago et al. (2016), and no systematic difference was found. 


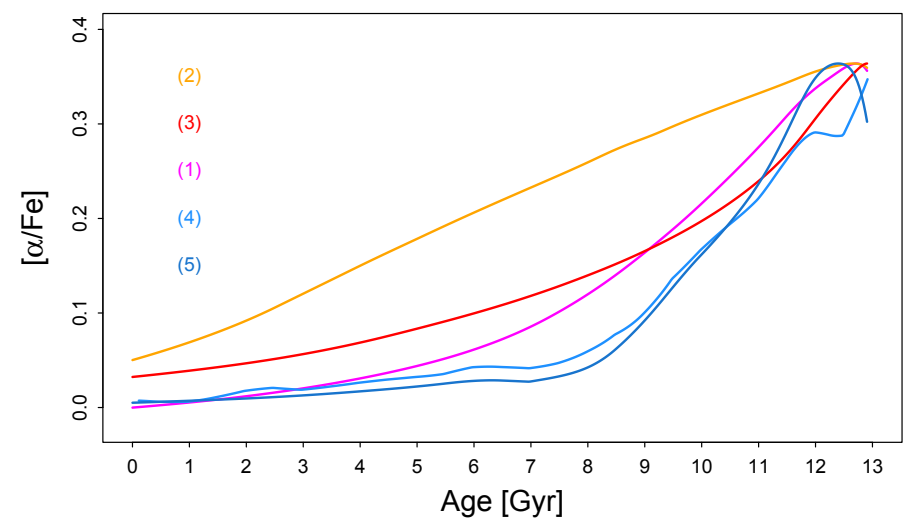

Fig. 5. Age- $[\alpha / \mathrm{Fe}]$ relations generated by the various SFHs of Fig. 4a. Numbers correspond to the SFHs of Fig. $4 \mathrm{a}$. The slope of the age- $[\alpha / \mathrm{Fe}]$ relation at a given age is proportional to the intensity of the SFR at this age.

to the low- $\alpha$ sequence are essentially discarded by the selection, and the Sun itself would not be selected. The model track in Fig. 3 shows that the inner disk had already reached solar metallicity 9 Gyr ago, or roughly 4 Gyr before the solar vicinity. This means that solar vicinity stars, or OLR stars, have followed a slightly different chemical evolution history than innerdisk stars. Hence, although the quenching episode we discuss in the present study occurred when the inner disk had reached solar metallicity, solar vicinity stars of solar metallicity were not affected because they formed $\sim 5$ Gyr later.

Therefore, stars selected in the solar vicinity (Fig. 3b) are likely to be contaminated by objects born at the interface between the inner- and outer-disk stars that are not truly members of the inner disk, such as the Sun. Histograms on the right show the number of selected stars in bins of $[\alpha / \mathrm{Fe}]$ once the outer disk is removed. They all show a bimodal distribution with a minimum between 0.17 and 0.07 dex. We emphasize that the minimum does not separate the thick disk from the thin disk, which were defined as those stars that make the low- $\alpha$ sequence. The minimum is a variation of stellar densities along the inner-disk sequence and separates the thick disk from the thin disk, defined as stellar populations in the sense given in Haywood et al. (2013). In this sense, the two populations are defined by distinct segments in the age- $[\alpha / \mathrm{Fe}]$ relation, which have been shown in Snaith et al. (2015) to correspond to two distinct phases of star formation. With this definition, the thick and thin disks follow the blue track in Fig. 3, with the thin disk represented by the portion to the right of the dot on the blue track. At all distances from the Galactic centre, the $\alpha$ distribution is clearly bimodal and shows a clear dip between 0.07 and 0.17 dex.

It has been shown by Hayden et al. (2015) that the sampling in directions, magnitudes, and colours of APOGEE does not introduce any significant bias in the metallicity distribution function of the survey. Giants, however, probably bias the underlying age distribution against the oldest objects or the most $\alpha$-rich stars, see Bovy et al. (2014). Hence, we keep in mind that the peak at $[\alpha / \mathrm{Fe}] \sim+0.23$ dex possibly underestimates the relative number of old stars compared to younger ones.

\subsection{Model stellar densities: why is the $[\alpha / \mathrm{Fe}]$ distribution bimodal?}

The bimodality of the $[\alpha / \mathrm{Fe}]$ distribution has been discussed by others, see for instance Fuhrmann (2004), Schönrich \& Binney (2009), and Bovy et al. (2012a). We now show that a bimodal density distribution along the inner-disk sequence of $[\alpha / \mathrm{Fe}]$ requires a very specific form of the SFH. To illustrate this, we first calculate the expected density distributions in $[\alpha / \mathrm{Fe}]$ for a range of SFHs along with their corresponding density distributions (Fig. 4). The age scale shows the temporal evolution of the $[\alpha / \mathrm{Fe}]$ abundance ratio for the SFH 4 . Two results are worth noting. First, different SFHs lead to different minimum $[\alpha / \mathrm{Fe}]$ abundance ratios at the end of the evolution. SFH 2 for example, with a slowly rising SFR, produces only a limited number of SNIa at early times and therefore a limited amount of iron at later times, hence the $[\alpha / \mathrm{Fe}]$ stays high - reaching values slightly below 0.05 dex. This is compared to SFHs 1 and 4, which have an SFR sufficiently high at early times such that the iron subsequently generated decreases the $[\alpha / \mathrm{Fe}]$ ratio just below 0 . SFH 5 reaches even lower $[\alpha / \mathrm{Fe}]$ values because of a slightly higher SF intensity between ages of 12 and 10 Gyr. This is also illustrated in Fig. 5, which shows the age- $[\alpha / \mathrm{Fe}]$ relation for the various SFHs in Fig. 4.

Second (and most important) for SFHs 1, 2, and 3, the corresponding stellar densities reach a maximum, or near maximum density, in the interval where the data show a minimum density, between 0.05 and 0.17 dex in $[\alpha / \mathrm{Fe}]$. The SFH from Snaith et al. $(2014,2015)$ is plotted in Fig. $4 \mathrm{a}$ and the corresponding stellar densities as a function of $[\alpha / \mathrm{Fe}]$ in Fig. $4 \mathrm{~b}, \mathrm{SFH} 5$ is equivalent to SFH 4 (except for a higher intensity between 12 and $10 \mathrm{Gyr}$ ), with the noise smooth from the SFH (4).

Star formation histories 4 and 5 show that a sudden drop and cessation of the star formation activity between 7 and $8.5 \mathrm{Gyr}$ are able to generate the dip in the stellar densities observed in the data. Figure 4 demonstrates that the observed shape of the $[\alpha / \mathrm{Fe}]$ distribution requires a particular SFH. Because of the tight correlation between $[\alpha / \mathrm{Fe}]$ abundance ratio and stellar age, the drop in the SF activity must occur at a precise and well-constrained epoch.

The bimodality in the $\alpha$ distribution arises for two reasons. The first reason is the slow evolution of $[\alpha / \mathrm{Fe}]$ with age in the thin disk, which is reflected in the shallow slope of the age$[\alpha / \mathrm{Fe}]$ relation at $<7 \mathrm{Gyr}$ (see Fig. 5). For ages younger than this, the variation of $[\alpha / \mathrm{Fe}]$ with age becomes slow and stars accumulate around $[\alpha / \mathrm{Fe}] \sim+0.025 \mathrm{dex}$, as is the case for all SFHs of Fig. 4 except for SFH 1. The steeper slope of the age- $[\alpha / \mathrm{Fe}]$ relation in the thick-disk sequence has the effect of decreasing the number of stars per unit $[\alpha / \mathrm{Fe}]$. Together with the lull in the SFH at $8 \mathrm{Gyr}$, this generates the dip in the $[\alpha / \mathrm{Fe}]$ distribution between 0.07 and 0.14 dex. The stellar density rises again only towards higher $[\alpha / \mathrm{Fe}]$ as a result of the higher SFR during the thick disk phase, generating the bimodality that is seen in Figs. 3 and 6. This means that the bimodality (and the dip in between) is the result of both the SFH and the nature of the age- $[\alpha / \mathrm{Fe}]$ relation.

\subsection{Model-data comparisons}

Figure 6 shows both the stellar mass (a to d) and the stellar counts (e to h) as a function of $[\alpha / \mathrm{Fe}]$ or $[\alpha / \mathrm{M}]$ of all SFHs compared to the histograms of the stars belonging to the inner-disk sequence of the four datasets used to make Fig. 3. The stellar counts were evaluated by assuming a Kroupa (2001) initial mass function (IMF; the same as assumed in the chemical evolution model). At each age, we assumed that the mass is distributed according to the IMF along an isochrone at the metallicity given by the age-metallicity of the model. For each isochrone, we selected stars that, having $\log g<3$, are distributed along the giant branch. Admittedly, this is a rough procedure, but it aims to show that by taking the effect of the IMF and the expected 

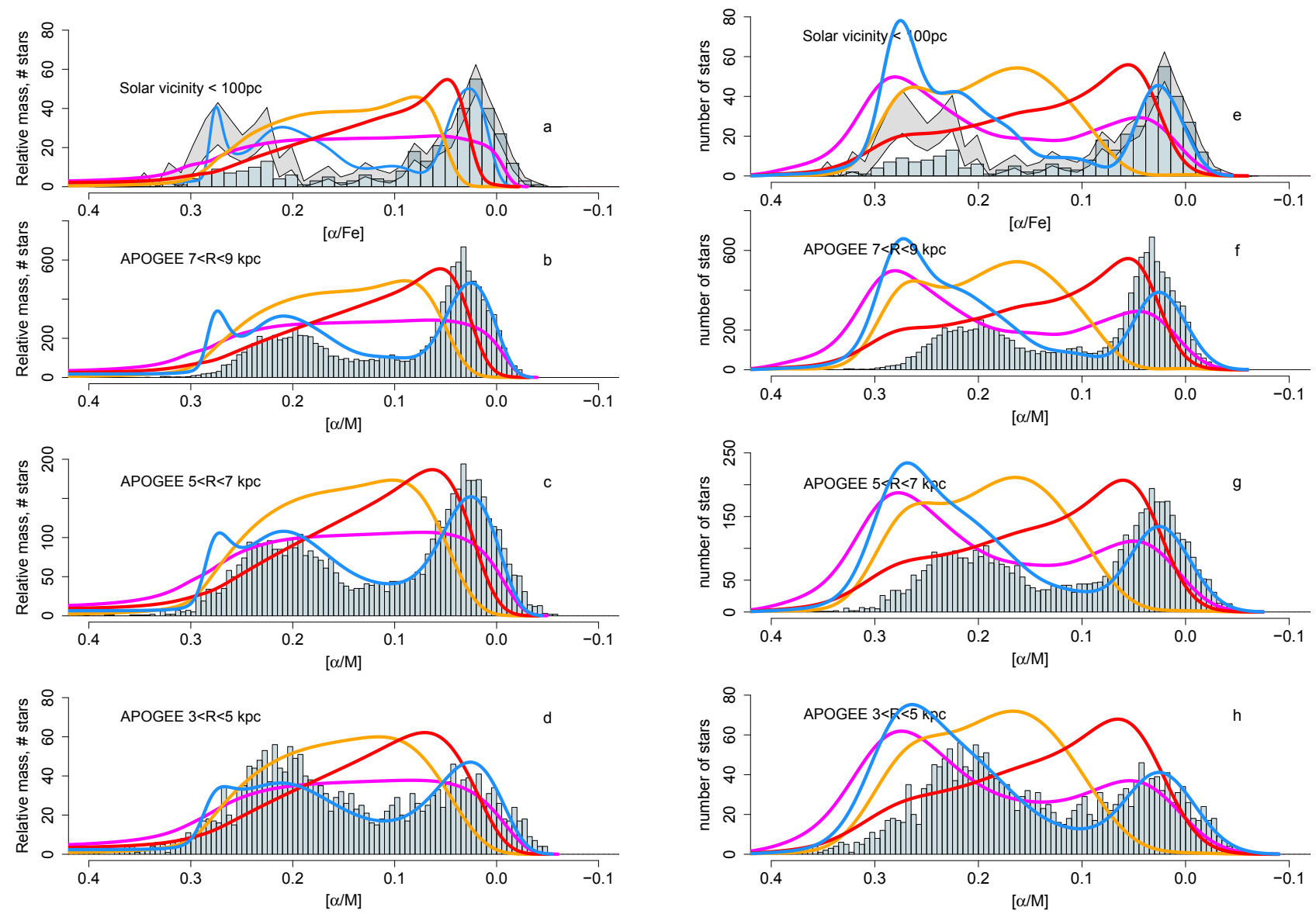

Fig. 6. a) Comparisons between observed and modelled mass (abcd) and star number (efgh) distributions as a function of $[\alpha / \mathrm{Fe}]$. Plots a) and e) show our calibration sample (solar vicinity) and panels (bcd) and (fgh) the APOGEE survey. The solar vicinity observed distribution in panels a) and $\mathbf{e}$ ) has been corrected to account for the lower sampling of old populations in the Galactic plane. From top to bottom, models have been convolved with increasing dispersion $(0.01,0.015,0.020$, and 0.025 dex $)$ to account for the difference in data quality. For comparison with APOGEE data, models have been shifted by +0.025 dex to allow for an offset in the $\alpha$-element abundance scale between our calibration sample and APOGEE.

distribution of stars along the giant branch into account, our results are essentially the same. Stellar counts (Figs. 6e-h) show distributions that are generally more heavily weighted in favour of $\alpha$-rich, older stars: younger giants, which are more massive, are less numerous, as expected from the IMF. This is particularly true for SFH 2. The consequence is that, at $[\alpha / \mathrm{M}]>0.2$ dex, even our best model lies significantly above the data. However, this may well be an effect of using giants as tracers, which, as mentioned in Sect. 3.1, may have biased the observed counts against $\alpha$-rich stars. We note that in spite of the crude conversion into counts and this possible bias, the bimodality of the distribution is preserved for SFH 4.

The data are confined to stars within the solar vicinity (distances within $100 \mathrm{pc}$ ) in Figs. 6a and e. This systematically underrepresents old populations, which are more widely dispersed throughout than younger populations of stars. We applied a correction to convert local stellar volume densities (grey histogram) into relative surface densities (grey area, which reflects corrected densities \pm Poisson uncertainties) using the result that monoabundance subcomponents are isothermal (Bovy et al. 2012c). The disk can therefore be modelled as a series of exponential subcomponents of different ages, or equivalently, $[\alpha / \mathrm{Fe}]$ abundances. Assuming exponential density laws in $z$, the corrections to relative surface densities only reflect the scale height of each subcomponent. Scale-height information for subcomponents in the $[\mathrm{Fe} / \mathrm{H}]-[\alpha / \mathrm{Fe}]$ plane were derived by Bovy et al. (2012b) from SEGUE. We used this information in the following way: $\alpha$-rich stars (on the SEGUE scale) with $[\mathrm{Fe} / \mathrm{H}]<-0.7$ dex have a scale height of $h_{z}=856 \mathrm{pc}$, while those with $[\mathrm{Fe} / \mathrm{H}]>$ -0.7 dex but $[\mathrm{Fe} / \mathrm{H}]<-0.3$ dex have $h_{z}=583$ pc. A metallicity of $[\mathrm{Fe} / \mathrm{H}] \sim-0.7$ dex along the inner-disk sequence corresponds to $[\alpha / \mathrm{Fe}] \sim 0.25$ dex on our scale, while a metallicity of $[\mathrm{Fe} / \mathrm{H}] \sim-0.3$ dex corresponds to $[\alpha / \mathrm{Fe}] \sim 0.2$ dex. Higher metallicities are divided into two groups: the $\alpha$-rich and $\alpha$-poor $([\alpha / \mathrm{Fe}]<0.10-0.15 \mathrm{dex})$, with scale heights of $348 \mathrm{pc}$ and $239 \mathrm{pc}$. Normalized to this last scale height, the corrections are $1.00,1.45,2.44$, and 3.58 applied in the intervals of $[\alpha / \mathrm{Fe}]<0.10,0.15<[\alpha / \mathrm{Fe}]<0.20,0.20<[\alpha / \mathrm{Fe}]<0.25$, and $[\alpha / \mathrm{Fe}]>0.25$ dex. The correction to surface densities enhances the densities between 0.2 and 0.3 dex, slightly shifted by $\sim 0.05$ dex compared to the model, but this is expected given that the scale heights from SEGUE were mapped on a $[\mathrm{Fe} / \mathrm{H}]-$ $[\alpha / \mathrm{Fe}]$, which is significantly different from the abundances in APOGEE. However, the level of the high- $\alpha$ peak is correct.

Figures $6 \mathrm{~b}$ and $\mathrm{f}$ shows the APOGEE data at the solar cylinder (7 to $9 \mathrm{kpc}$ from the Galactic centre) for stars with a signalto-noise ratio $(\mathrm{S} / \mathrm{N})$ greater than 150 . To ensure that frequency distributions were not affected by the exact choice of $\mathrm{S} / \mathrm{N}$, we checked that this only changes the relative significance of the peaks by $<10 \%$. Old populations at large distances from the 
Galactic plane are much better sampled in these survey data than in our reference sample (Fig. 6a). The drop in and cessation of the star formation activity for $\sim 1$ Gyr leads to a strongly bimodal distribution that is well reproduced by our model SFH. Figure 6, panels $\mathrm{c}$ and $\mathrm{g}$ and $\mathrm{d}$ and $\mathrm{h}$, shows the distributions outside the solar vicinity for the distance range 5 to $7 \mathrm{kpc}$ and 3 to $5 \mathrm{kpc}$ from the Galactic centre. For this analysis, we adopted a less stringent $\mathrm{S} / \mathrm{N}$ cut (only 80 , compared to 150 used in the previous sample) to allow for a larger number of stars and better statistical sampling of all populations, especially the older, generally more distant stars. All plots illustrate that SFHs 1, 2, and 3 are incompatible with the observed stellar densities. None of these SFHs is able to reproduce the dip in stellar densities between $0.07<[\alpha / \mathrm{Fe}]<0.17$ dex. In contrast, SFH 4, with a sharp drop between 10 and $9 \mathrm{Gyr}$ and no or little star formation between 9 to $7.5 \mathrm{Gyr}$, agrees remarkably well with both the local and the remote $[\alpha / \mathrm{Fe}]$ distributions, reproducing both the peak centred on $[\alpha / \mathrm{Fe}]=0.02$ dex and the sharp decrease at $[\alpha / \mathrm{Fe}] \sim 0.05$ dex. SFH 4 explains the distribution remarkably well considering it was not tuned in any way to fit these data, but is precisely the SFH derived by fitting the evolution of $[\mathrm{Si} / \mathrm{Fe}]$ versus time using local data (Snaith et al. 2014, 2015). For SFH 2 and 3, the dip is not reproduced, but neither is the peak of stars at $0.025 \mathrm{dex}$ (thin disk) because of the effect mentioned in the previous section: the number of SNIa produced early in the thick-disk phase in these models is small because the SFR during this period is low and the iron ejected by SNIa is limited, which then affects the decrease rate of $[\alpha / \mathrm{Fe}]$.

We conclude these comparisons by emphasizing the following important point: the dearth of stars in the SFH between 7 and 8.5 Gyr was detected using two entirely different approaches. The SFH from Snaith et al., with a dip at $\sim 8 \mathrm{Gyr}$, was derived from fitting the shape of the relation of $[\alpha / \mathrm{Fe}]$ abundance vs. age alone, and in this case, observed stellar densities played no role. As shown in Snaith et al. (2015), the (abrupt) change in the slope of $[\alpha / \mathrm{Fe}]$ abundance vs. age requires the dip in the SFH to reproduce the $[\alpha / \mathrm{Fe}]$ abundance vs. age data. Stellar densities from APOGEE now provide new and crucial evidence of a rapid change in the star formation intensity between the two epochs of thick- and thin-disk formation. We show below that the dip in the SFH has also been found in local age distributions of stars (see Sect. 3.7), although it has not always been recognized as such.

\subsection{Effect of the IMF}

The IMF enters our comparisons in two ways. The first is through the chemical evolution model, the second is through computing the number of stars as a function of $[\alpha / \mathrm{Fe}]$. We consider each of these in turn. The IMF is an input of the model that was used in Snaith et al. (2015) to adjust the observed age$[\mathrm{Si} / \mathrm{Fe}]$ relation by varying the $\mathrm{SFH}$. Hence, changing the IMF affects the resulting best-fit SFH to the chemical evolution of the MW. It was shown in Snaith et al., however, that the differences are small for a reasonable range of IMFs. This is illustrated in Fig. 14a of Snaith et al., which shows that the SFHs obtained for the Kroupa, Salpeter and Scalo IMFs all show a significant dip at $\sim 8$ Gyr. Only the IMF from Baldry \& Glazebrook (2003) can reproduce the age-[Si/Fe] relation without a dip in the resulting SFH. However, the model generated with the IMF from Baldry \& Glazebrook (2003) significantly overestimates the $[\mathrm{Si} / \mathrm{Fe}]$ abundances at all metallicities. The best-fit model using the Scalo IMF produces a dip in the SFH, but also significantly modifies the ratio of thin to thick disks. Again, however, the model with a Scalo IMF results in a significant offset of the

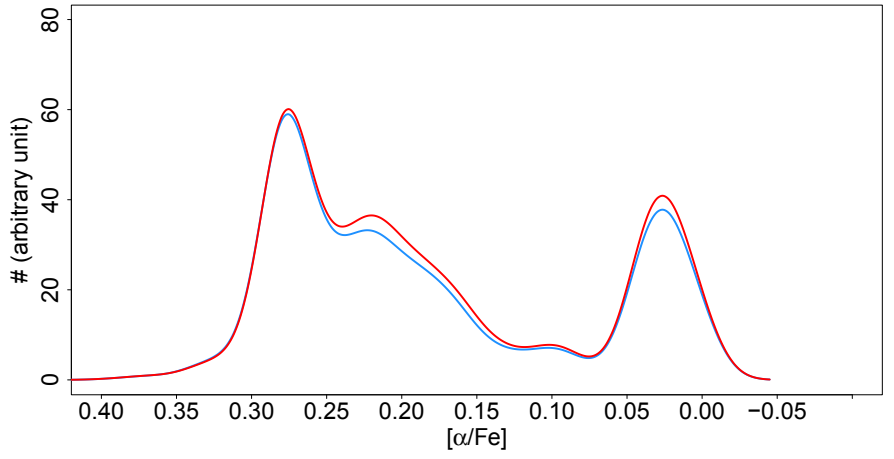

Fig. 7. Effect of the IMF on the relative mass distribution.The blue curve is derived using a Kroupa (2001) IMF, the red curve with a Salpeter IMF.

inner-disk $[\mathrm{Fe} / \mathrm{H}]-[\alpha / \mathrm{Fe}]$ sequence by underestimating $[\mathrm{Si} / \mathrm{Fe}]$ by about 0.05 dex at a given metallicity. We find that only the Kroupa IMF enables us to fit all constraints given by elemental abundances of stars within the solar vicinity.

We now consider how the functional form of the IMF shapes the observed and modelled stellar density distributions as a function of $[\alpha / \mathrm{Fe}]$. The effect of the IMF is likely to be significant if the range of masses that enters the selected stars of Fig. 6 is large. In this case, the IMF could have an effect on the ratio of the most massive to least massive stars in the distribution. However, this is unlikely to be the case: the mass range of giants that enter the sample is rather small, limited to about $0.4 M_{\odot}$. Figure 7 shows that the effect is small, with the two curves obtained assuming a Kroupa (2001) and a Salpeter IMF (blue and red curves).

\subsection{Radial migration}

What are the possible effects of radial migration on the present work? Our analysis relies on a clearly identifiable inner-disk sequence in both the Adibekyan et al. data and the APOGEE survey. We recall that by inner disk, we mean both the thin- and the thick- disks stars that make the clear - and almost uncontaminated - sequence visible in the APOGEE data at $R<7 \mathrm{kpc}$ in the $[\mathrm{Fe} / \mathrm{H}]-[\alpha / \mathrm{Fe}]$ plane. This sequence is also visible in the solar vicinity, but in continuity with the low- $[\alpha / \mathrm{Fe}]$ sequence. The outer disk $(R>10 \mathrm{kpc})$ contains no or very little inner-disk stars and results from a different chemical evolution according to our description in Snaith et al. (2015). Haywood et al. (2013, 2015) argued that the tight relations between age and metallicity (in the thick-disk phase) and $\alpha$-abundances for stars on the inner-disk sequence observed in the solar vicinity imply that the chemical evolution and star formation in the inner disk must have been remarkably homogeneous and uniform. This is now confirmed by the similar chemical patterns seen in APOGEE at different Galactocentric distances (see Hayden et al. 2015, and Fig. 3). It may be that, as noted in Hallé et al. (2015), radial migration has mixed the inner disk inside the OLR, but mixing stars that have uniform properties, independent of Galactocentric distance, will not change any observed trend.

More generally, Fig. 4 of Hayden et al. (2015) shows that the inner-disk sequence clearly dominates at $R<7 \mathrm{kpc}$ and the outer-disk sequence at $R>10 \mathrm{kpc}$, with a limited overlap of the two outside the $7-9 \mathrm{kpc}$ ring and almost none outside the 7-11 kpc ring: chemical patterns at $R<7 \mathrm{kpc}$ are essentially different from those at $R>11 \mathrm{kpc}$. This confirms that the solar vicinity is a transition zone between the inner and outer disk, as 
anticipated in Haywood et al. (2013). In this same article, we concluded that radial migration must have been very limited and cannot "have redistributed stars in significant proportion across the solar annulus", which is what is observed in APOGEE. These results favour models where the evolution of inner and outer disk are disconnected. By extension, they exclude models with extensive radial migration, where large portions of stars at a given radius come from large distances (more than $2 \mathrm{kpc}$ ) and where inner and outer disks have similar chemical patterns because they result from the same chemical evolutionary history (see e.g. Minchev et al. 2014). Hence, radial migration probably plays essentially no significant role in the distribution of $\alpha$-abundance analysed here.

\subsection{Possible biases}

Using the SEGUE survey, Bovy et al. (2012a) found that three effects may conspire to produce an artificial bimodality in the star count distribution of $[\alpha / \mathrm{Fe}]$ abundances: (1) the selection function of the survey; (2) counting stars in volume rather than considering surface densities; and (3) the binning of stars per unit $[\alpha / \mathrm{Fe}]$ instead of mass per unit $[\alpha / \mathrm{Fe}]$, because lower metallicity stars have lower masses, hence the metal-poor peak represents relatively less mass than the metal-rich peak. According to Bovy et al. (2012a), this last effect is likely to be small because the difference in mass between $[\mathrm{Fe} / \mathrm{H}]=-1.5$ and 0.3 dex is $\sim 20 \%$. Figure 3 shows that the two peaks cover an even smaller range, being separated by $\sim+0.5$ dex, which means that the effect is likely to be significantly smaller than even this estimate.

We now consider points (1) and (2).

(1) The selection function of the survey. Hayden et al. (2015) have shown that the sampling function of APOGEE does not introduce any significant bias in the metallicity distribution functions (MDF). Although there might be a bias in the $[\alpha / \mathrm{Fe}]$ distribution without producing a bias in the $[\mathrm{Fe} / \mathrm{H}]$ distribution, we note that the MDF of APOGEE in the inner disk is clearly bimodal, with one peak at +0.25 and another at -0.25 dex. Since the MDF is not biased by the selection function of APOGEE, we considered this as a real astrophysical feature. Because of the good correlation of $[\alpha / \mathrm{Fe}]$ with metallicity within the inner-disk sequence, it is expected that a bimodality in $[\mathrm{Fe} / \mathrm{H}]$ distribution directly corresponds to a bimodality in the $[\alpha / \mathrm{Fe}]$ distribution.

We note that if the selection function of SEGUE were responsible for producing the bimodality, we would not expect this feature to arise in other surveys, but it is present in both APOGEE and GES (see Recio-Blanco et al. 2014). We note also that in the case of solar vicinity samples, the selection function of large surveys does not enter into consideration, but the bimodality is still present.

(2) It might be that this is an effect of the volume sampling (i.e. counting stars in volume rather than considering surface densities). Converting volume densities into surface densities might alleviate the bimodality. Volume sampling of stars in the solar vicinity shows the bimodality. If the conversion into surface densities were able to alleviate the bimodality, it would imply that stars with intermediate $[\alpha / \mathrm{Fe}]$ abundances (corresponding to the gap) are more numerous at larger heights than they are close to the Galactic plane. However, at all distances from the Galactic plane, the distribution of $[\alpha / \mathrm{Fe}]$ abundance is either bimodal or dominated by stars that belong to either the $\alpha$-poor or $\alpha$-rich peaks of the distribution. This is illustrated very well in Fig. 8, which
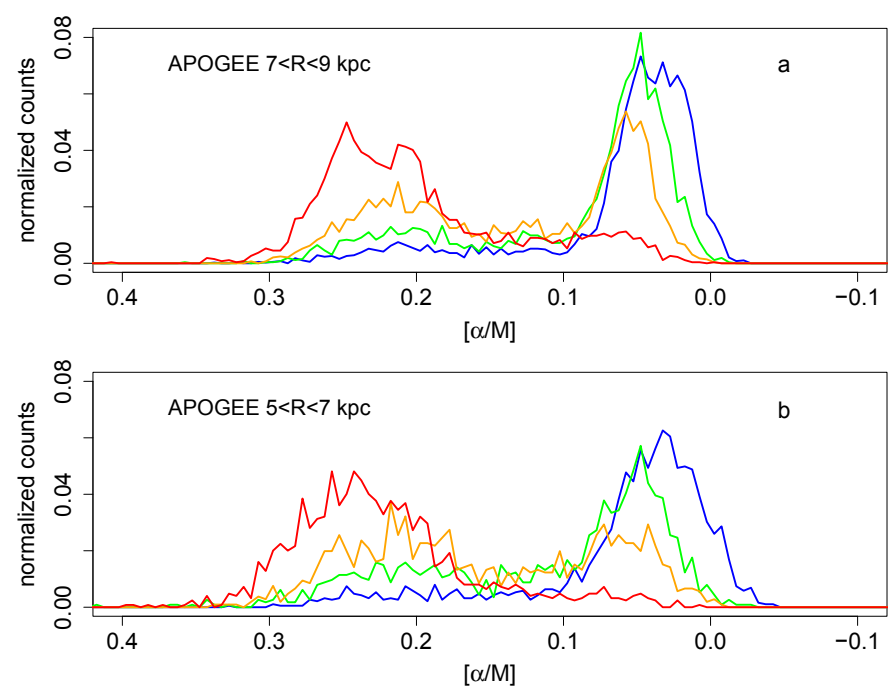

Fig. 8. Normalized $[\alpha / \mathrm{M}]$ counts from APOGEE in two different distance bins to the Galactic centre (the distances are indicated in each plot). The different curves correspond to different $z$ intervals above the Galactic plane. Red represents stars above $1 \mathrm{kpc}$, orange those between 1 and $0.5 \mathrm{kpc}$, green stars between 0.5 and $0.25 \mathrm{kpc}$, and blue stars at $0.25 \mathrm{kpc}$ relative to the Galactic mid-plane. At no height above the Galactic plane are the star counts dominated by stars with $[\alpha / \mathrm{M}]$ between 0.08 and 0.18 dex.

shows the normalized counts of stars selected as explained in Sect. 3.1 (and which lie above the black line in Fig. 3) as a function of $[\alpha / \mathrm{M}]$ at different heights above the Galactic plane, $0-0.25,0.25-0.5,0.5-1.0$, and above $1 \mathrm{kpc}$. It shows that stars with $[\alpha / \mathrm{M}]$ in the range $(0.08,0.18)$ dex are not dominant at any height. Hence, there is no reason to expect that by simply summing the contributions of stars at all disk heights we would fill the gap.

Beyond these considerations, some additional comments are warranted. First, abundance determinations are more precise in APOGEE than in SEGUE because of the higher spectral resolution and $\mathrm{S} / \mathrm{N}$ of its data, which enhance the contrast between the peaks and the gap of the distribution in APOGEE (cf., for instance Fig. 1 left of Bovy et al. 2012a, and Fig. 6 shown here). Second, in estimating the $[\alpha / \mathrm{Fe}]$ abundance distribution, Bovy et al. selected all stars in the $([\mathrm{Fe} / \mathrm{H}],[\alpha / \mathrm{Fe}])$ plane, including stars within the metal-poor thin disk, which in the SEGUE survey do not appear as a separate sequence because of the lower spectral resolution and poorer determinations of the metallicities. The metal-poor thin-disk stars, however, represent the separate chemical evolutionary history of the outer disk (see Haywood et al. 2008, 2013; Snaith et al. 2015). The oldest and most metal-poor of these stars have $[\alpha / \mathrm{Fe}]$ abundances intermediate between the $\alpha$-rich (old thick-disk) and $\alpha$-poor (thin-disk) stars. Although such metal-poor outer-disk stars have similar $\alpha$ abundances, they have metallicities well below that of the thick disk at the same age (Haywood et al. 2013) and therefore would contribute to filling in the gap in the stellar abundance distributions of the inner-disk stars. Hence, in attempting to understand the evolutionary history of the inner disk, these stars should not be mixed into the analysis (as we emphasized several times in this article). 


\subsection{Previous findings}

The SFH derived in Snaith et al. $(2014,2015)$ is the first, and up to now, the only, determination of the general SFH of the MW. All previous available studies were determinations of the local age distributions. It is important to emphasize that age distributions determined from local data are not SFHs of the MW, although they sometimes are inaccurately referred to as such. The contribution of thick-disk stars, in particular, are underestimated because while scale-height corrections are sometimes applied to take the contribution of older stars at larger distances from the Galactic plane into account, the thick disk lies predominantly in the inner Galaxy and its contribution in the solar vicinity is small. The SFH derived from abundances assumes a model to fit the age-abundance relation, but no density correction is needed. The derived SFH is general and representative of the whole disk as far as the age- $[\alpha / \mathrm{Fe}]$ abundance relation is general and representative. If so, it means that the minimum we detected in the SFH in chemical data in Snaith et al. (2015) and now in star count data (Sect. 3.3) should also be present in local age distributions.

Cignoni et al. (2006) determined an age distribution from the HIPPARCos HR diagram. They found a distribution that shows a broad minimum between ages of 6 to $10 \mathrm{Gyr}$, showing an upturn in the last bin between 10 and 12 Gyr. Because the resolution in age that can be obtained from inverting the HR diagram for old stars is poor and because thick-disk stars are poorly represented in the solar vicinity, studies of this type provide little information about the detail of the age distribution of thick-disk stars. In addition, as just mentioned, this population is strongly underrepresented if volume corrections are not applied, as is the case in this particular study. However, because the corrections are expected to be significant only for the oldest stars $(>9-10 \mathrm{Gyr})$, the dip found by Cignoni et al. (2006) would remain, in agreement with our general findings.

Gratton et al. (1996; see also Reddy et al. 2006) were probably the first to suggest from the analysis of chemical abundances the possibility of a gap in the SFH of the MW, at the transition of the thick to the thin disk. Bernkopf \& Fuhrmann (2006) and Fuhrmann (2011) suggested that an age gap of about 4 Gyr separates the thick and thin disks, having found that the thick disk is a $12 \mathrm{Gyr}$ old burst population, with only few stars with intermediate $(\sim 10 \mathrm{Gyr})$ age. These findings are roughly compatible with our own results, excluding the fact that the thick disk is a burst population, as already mentioned in the introduction.

Rowell (2013) used the white dwarf luminosity function to investigate the SFH in the past $9 \mathrm{Gyr}$. He found that the SFH is bimodal with two broad peaks at 2-3 Gyr and 7-9 Gyr, separated by "a significant lull of magnitude 30-90\% depending on the choice of the cooling models". Adopting the cooling models of Fontaine et al. (2001), the estimated SFH shows a very significant pause extending from 5 to $7 \mathrm{Gyr}$ with a minimum at $\sim 6 \mathrm{Gyr}$, compatible with zero star formation activity. As was also the case for Cignoni et al. (2006), Rowell (2013) determined an age distribution of stars in the solar vicinity. Similarly, a true SFH would require volume corrections that were not applied. Here again, the most significant corrections would apply to thick-disk stars. Hence, if these corrections were taken into account, the contrast between the SFR at ages $>7$ Gyr and the SFR during the minimum would be increased. The lull detected by Rowell would remain, and this lull again would generally agree with our favoured SFH. Another significant difference is the fact that the minimum detected by Rowell (2013) occurs at $6 \mathrm{Gyr}$, while in our case the SFH is minimum at $\sim 8 \mathrm{Gyr}$.
This is not as critical as it seems, however, because there are still large differences between isochrone datings and ages deduced from white dwarfs. For instance, Fontaine et al. (2001) estimated the age of the disk to be of the order of $11 \mathrm{Gyr}$, or at least $2 \mathrm{Gyr}$ younger than the age we determined for the oldest thick-disk stars (Haywood et al. 2013). Kalirai (2012, see also Kilic et al. 2010) measured an age for the white dwarfs from the Galactic halo of $11.4 \pm 0.7 \mathrm{Gyr}$, significantly younger than local halo subdwarfs (see for example VandenBerg et al. 2014, who derived ages of $12 \pm 0.14,12.56 \pm 0.46$, and $14.27 \pm 0.38$ for three local subgiants). Similarly, ages of globular clusters derived from white dwarfs can also be younger than those obtained from isochrone fitting technics: for instance, Hansen et al. (2007) derived an age for NGC 6397 of $11.47 \pm 0.47 \mathrm{Gyr}$, while Gratton et al. (2003) for instance derived an age of $13.9 \pm 1.1 \mathrm{Gyr}$ (see comments in Torres et al. 2015).

The conclusion from these comparisons is that using three entirely different datasets and methods, three studies found evidence of a significant dip in the age distribution of stars in the solar vicinity, supporting our general findings.

\section{Discussion}

The data from APOGEE shown in the previous section include stars from 3 to $9 \mathrm{kpc}$ from the Galactic centre and demonstrate that the dip found in the SFH is a pervasive feature that occurs throughout the disk. This implies, unlike any other previous result or analysis, that the cessation of the star formation activity was a general phenomenon that did not only occur at the solar cylinder. Remarkably, in less than $2 \mathrm{Gyr}$, from 10 to $8.5 \mathrm{Gyr}$ ago, the star formation rate has decreased by more than a factor of 10 . At a redshift of around 1, the MW was on its way to become a lenticular galaxy (see also Sil'chenko 2013), and then the star formation activity resumed (see Sect. 4.4 for a possible explanation). We now discuss the consequences of this finding.

\subsection{Thick and thin disks: are they the same population?}

Recently, Bovy et al. (2012a,b) has discussed the possible continuity between thick- and thin-disk stars. Their arguments were twofold. They first concluded that the dip in the $[\alpha / \mathrm{Fe}]$ distribution is only apparent and not due to astrophysical causes, but is instead due to selection and volume effects. This conclusion is not confirmed by our analysis, as we explained in Sect. 3.2. The second argument is based on the measurement of structural parameters of mono-abundance populations from star counts, which shows a smooth transition in scale height and scale length from thick- to thin-disk stars. This analysis has been confirmed from APOGEE data by Bovy et al. (2015). We return to this argument below, but we first wish to add two important facts that must be taken into account in this debate. It was suggested three decades ago that the thick disk pre-enriched the thin disk (Gilmore \& Wyse 1986). Indeed, although there is a break in the slope of the age- $[\alpha / \mathrm{Fe}]$ relation that clearly points to two separate epochs of star formation, there is strict continuity in the chemical properties of the two populations (as shown in Fig. 6 of Haywood et al. 2013; and Fig. 3 of Haywood et al. 2015): the thin inner disk originally inherited the chemical traits of the thick disk. The change of slope in the age- $[\alpha / \mathrm{Fe}]$ relation is due to a change of the intensity of the star formation between the two epochs. The present findings now confirm that the transition between the two disks probably occurred during a period of almost complete cessation of the star formation activity. Hence, 
although there is continuity in elemental abundances and possibly in structural properties, the two populations are well differentiated in their SFHs: the thick and thin disks formed their stars with two distinct phases of Galactic evolution.

How can we account for the results showing a continuity in the structural parameters under these circumstances? The thickdisk population cannot be described with a single scale height. The analysis of Bovy et al. (2012b, 2015) showed that the $\alpha$-rich population can be decomposed into a series of subpopulations whose scale height decreases with increasing metallicity. This is confirmed by data from stars in the solar vicinity, where it has been found that the vertical velocity dispersion continuously decreases with age within the thick disk (Haywood et al. 2013, Fig. 11). This means that although the gap in time between the start of one population and the end of the other was relatively large, any discontinuity left in the structural parameters of these two populations is probably small simply because the structural properties of the young thick disk and the old thin disk are very similar. In addition, various dynamical effects may have blurred possible differences in kinematics and spatial distributions of the populations of the oldest thin-disk and the youngest thick-disk stars over the past 8 Gyr.

In view of these results, the discussion of a continuity (or not) between the two populations, based on a decomposition of structural parameters of "mono-abundance subpopulation" appears contrived. The use of structural parameters as defining properties of a stellar population is implicit in the definition of all MW stellar populations, which are often viewed as spatial entities ("disk", "bulge", "halo"). But can we define a continuity between two populations solely on the basis of their structural parameters? The MW has a "bulge", but possibly no separate bulge population of its own. Recent studies such as those by Shen et al. (2010), Kunder et al. (2012), and Di Matteo et al. $(2014,2015)$ suggest that the bulge is basically a structure formed from disk stars through dynamical disk instabilities, a "pseudo-bulge". Hence, defining a population from its structural parameters is ambiguous at best. More accurately, a stellar population ought to be defined by parameters that are linked as directly as possible to the ISM out of which its stars formed and at the time they were formed. From this point of view, the thick and thin disks are clearly separate but parent or child populations.

\subsection{Star formation quenching in the Milky Way vs. other galaxies}

We interpret the decrease in the star formation activity in the MW 10 Gyr ago $(z \sim 2)$ and the lull in the SFR at 7-8.5 Gyr as a manifestation of the quenching. Quenching is observed in distant galaxies and has several defining characteristics. First, quenching in the MW is extended in time and represents a significant decline in the SFR by more than an order of magnitude in less than a Gyr, and this insignificant level of SF is maintained for about 1 Gyr. This agrees well with the observed quenching of MW-type galaxies in the early Universe (Morishita et al. 2015). Morishita et al. selected Milky Way-type progenitors at different redshifts using the technique of abundance matching where they studied the redshift evolution of star-forming galaxies with co-moving densities similar to MW-type galaxies in the local volume. They observed that the inner parts of the galaxies they selected statistically become redder at $z \sim 1.6$ and then quench their star formation at $z \sim 1.0$, in very good agreement with our time-line for the star-formation rates of the MW. We emphasize that a difference must be made here between quenching of the star formation activity and the transition from an active to a quiescent phase of star formation. Schawinski et al. (2014) for instance argued that quenching in late-type spirals is the slow transition from an actively star-forming galaxy to one that is quiescent as a result of the exhaustion of its gas. Clearly, the quenching observed in the inner disk of the MW is not a slow transition to quiescence. The drop in the SF activity in the MW was fast - less than $1 \mathrm{Gyr}$, but could be significantly more rapid than that since this is below the time resolution of our SFH. This means that while the transition to quiescence in the MW was fast, just as it appears to be in early-type massive galaxies, the MW is in strong contrast to early-type galaxies because its quenching phase was followed by the re-initiation of its star formation. This re-initiation of star formation lasted until today, formed roughly $50 \%$ of the MW's stellar mass, and the starformation rate was roughly constant. Between 9 and $7 \mathrm{Gyr}$, it is possible that the only significant SF activity occurred in the outer parts of the disk ( $R>10 \mathrm{kpc}$, see Haywood et al. 2013). However, because of the low gas and stellar densities in these outer regions, the star formation efficiency was likely very low and the integrated contribution of such star formation in the outer disk must have been small.

Phenomenologically and in particular, quenching in galaxies is observed through their bimodal distribution in colour-magnitude planes, whereby the quiescent, quenched galaxies form a red sequence, while the still actively starforming galaxies form a blue cloud (see e.g. Gabor et al. 2010, for a nice summary). The present position of the MW, whether in the blue cloud, in the green valley, or in between them, is unknown, essentially because of the uncertainties that plague the colour measurements of our Galaxy (Mutch et al. 2011; or more recently Licquia et al. 2015). It is possible, however, to comment on the possible track between the blue and red sequences of the MW at the time of its quenching. During the period of the thick-disk formation, the Galaxy was actively forming stars and therefore belonged to the blue cloud. The duration of the passage from the blue cloud to the red sequence depends on the quenching rate experienced by a galaxy. The faster the quenching, the faster a galaxy crosses the green valley. For a decrease of the SFR by an order of magnitude in 2 Gyr, as indicated by Fig. 2, the quenching timescale is $\sim 0.86 \mathrm{Gyr}$, which would correspond to a crossing time of $\sim 1$ Gyr (Martin et al. 2007). This means that at the end of the quenching phase, the MW was at the limit of the red sequence. Similar estimates from Blanton (2006) are that a sharp cut-off in star formation induces a migration to the red sequence in $\sim 1$ Gyr. The SFH of the MW is consistent with this.

The second reason to consider the observed lull in the SFH as a manifestation of quenching is that it is associated with a morphological transformation, marking the transition from the thick to the thin disk. Hence, it is not any fluctuation of the $\mathrm{SFH}$, but one that corresponds to a major phase in the history of the Galaxy, clearly reminiscent of the transitional phase that is detected in galaxies at high redshifts. A crucial difference to the standard interpretation is, however, that the quenching in the MW is linked to the thick disk, not to the bulge. The MW is most probably a pure disk galaxy (see e.g. Shen et al. 2010; Di Matteo et al. 2014, 2015). That is, its classical bulge, if it exists, is small $(\mathrm{B} / \mathrm{T}<0.1)$. In contrast, its thick disk is massive, totaling about half the stellar mass of the entire disk. We argue in the next section that bulge quenching, observed to take place in high-redshift samples, may often be thick-disk quenching instead.

We note that the quenching episode is also contemporaneous with other events in the MW. According to Haywood et al. (2013), the outer disk ignited star formation at 10 Gyr. The 
time at which the SF starts to slow down in the inner disk therefore coincides with the time when star formation activity ignites in the outer disk. The minimum of the star formation (at $z \sim 1$ ) may also be contemporaneous with the birth of the bar. Although the precise time of the bar formation is not known, galaxies of the mass of the MW are thought to form their bar at $z \sim 1$ (Sheth et al. 2008; Melvin et al. 2014). Interestingly, this is also the epoch at which Morishita et al. (2015) detected an increase in stellar mass in the central regions of MW progenitors, at a time when their galaxies have already quenched their star formation activity. Bars have been proposed as a possible agent of quenching in late-type galaxies (e.g. Gavazzi et al. 2015).

Another interesting feature from Morishita et al. (2015) is that they clearly observed that quenching occurs in the inner regions, while apparently SF continues in the outer parts. This is also compatible with the sequence of events described here for the MW, when the star formation activity shuts down in the inner regions while it ignites in the outer disk.

\subsection{Are these results compatible with the general history of late-type galaxies?}

The quenching episode measured here shows that galaxies like the MW may have had large variations of their SFR in their past, with a decrease by an order of magnitude occurring on a timescale of $\sim 1$ Gyr. The question is whether this variation is consistent with the observed dispersion of the main sequence of galaxies. Comparisons of our SFH with the main sequence have been presented in Lehnert et al. (2014), who showed that the MW lies systematically below the star formation rate that would be expected for a typical galaxy of its mass. Figure 2 of Whitaker et al. (2015), for example, shows all galaxies plotted in the main-sequence plane (either active or quiescent). It is evident there that with a star formation rate of a few solar masses per year and because of its own mass, the MW has been a quiescent, or almost quiescent, galaxy since $z<1$. This is compatible with its position in the green valley (see Mutch et al. 2011; Licquia et al. 2015).

In the thick-disk formation phase, our Galaxy had its most intense star formation activity, but also lay below the main sequence. We note that the MW is not an exception in this regard: for MW progenitors, van Dokkum et al. (2013) measured a star formation activity similar with what we measured for our Galaxy.

Assuming a total current stellar mass of $5 \times 10^{10} M_{\odot}$, the MW sustained an SFR of between 10 and $15 M_{\odot} \mathrm{yr}^{-1}$ during the thick-disk formation (see Fig. 1), and $10 \mathrm{Gyr}$ ago $(z \sim 2)$, the MW was half way to building its thick disk (with a stellar mass of the order of 1 to $1.5 \times 10^{10} M_{\odot}$ ). For a galaxy of this mass, the main sequence at redshift 2 is nearer to $30-40 M_{\odot} \mathrm{yr}^{-1}$. However, at a rate of $40 M_{\odot} \mathrm{yr}^{-1}$, the MW would have formed all its mass within $1.3 \mathrm{Gyr}$, which implies that it cannot have sustained this star formation activity for very long. A bursty mode that, while keeping a mean SFR of $10-15 M_{\odot} \mathrm{yr}^{-1}$, alternates short phases of intense star formation with more quiescent periods is not favoured, however, because it would probably inject sporadically high levels of metals in the ISM and would produce a dispersion in abundances that is not observed (of course unless the metal loss was tuned to keep the dispersion in the metallicities of the stars low), with the $[\mathrm{Fe} / \mathrm{H}]-[\alpha / \mathrm{Fe}]$, the age- $[\alpha / \mathrm{Fe}]$ and age-metallicity relations all being tight during the thick-disk phase (Haywood et al. 2013). This low dispersion of abundances and metallicities of stars at constant age is most simply explained by the MW having kept an approximately constant rate of star formation of $10-15 M_{\odot} \mathrm{yr}^{-1}$ during the phase of thickdisk growth. All this implies that our Galaxy, and possibly most galaxies of its type, has remained quiescent (in a relative way, this largely depends on the definition of the main sequence) for most of its history, possibly not lying along the ridge line that defines the main sequence of galaxies.

\subsection{What is the origin of this relatively brief quenched phase?}

In the particular case of the MW, it is difficult to associate the shutting down of star formation and its reactivation with a shortage of gas and then significant accretion of fresh fuel, since for such a scenario we would expect to see a corresponding signature in the evolution of the chemical abundances. In contrast, the age- $[\alpha / \mathrm{Fe}]$ relation shows a clear continuity (see Fig. 6 of Haywood et al. 2013, for example), which is very well represented by a closed-box model (Snaith et al. 2015). We therefore exclude the possibility that the quenching episode in the MW is directly tied to its history of the gas accretion.

Morphological quenching has been proposed by Martig et al. (2009) for galaxies with substantial spheroidal components, or thick disks, which could stop star formation by stabilizing the gas disk. At the end of the thick-disk phase, before the thin inner disk started to form, most of the MW stellar mass was located in a component which, with a scale length of $2 \mathrm{kpc}$ (Bensby et al. 2011; Cheng et al. 2012; Bovy et al. 2012b), was strongly concentrated in the inner regions. Although Martig et al. (2009) clearly envisaged that morphological quenching could apply to thick disks, we emphasized in Sect. 2.1 that at the end of the thick-disk phase, the gas fraction in the MW was still likely very high $(\sim 50 \%)$. This is far from the values of the case studied by Martig et al. (2009), where the gas fraction stays essentially below $15 \%$ throughout the transformation from disk to spheroiddominated galaxy. Hence, it is not clear how efficient morphological quenching would really be in the MW.

Other possibilities for quenching have been proposed more recently. For instance, it has been suggested that the heating of the gas from the radiation field of low-mass stars or the winds of dying low-mass stars could prevent star formation in galaxies, see Kajisawa et al. (2015) and Conroy et al. (2015). We also noted in the previous section that the formation of the bar in the MW is possibly contemporaneous to the quenching phase. Athanassoula et al. (2013) and Gavazzi et al. (2015) proposed that the bar might be responsible for the quenching of the star formation by sweeping the gas within the corotation radius to the central regions through the loss of angular momentum. While this is an attractive possibility, it is not clear in this scenario how to ensure that the star formation activity resumes to form the disk stars and - if it is through the accretion of fresh gas - how to ensure the chemical continuity between the thick- and thindisk stars. Moreover, in the scenario proposed by Gavazzi et al. (2015), the fraction of gas assumed is 5\% of the stellar mass, which is far from the case being considered here, where half the mass of the disk could still be in gas. While in the scenario of Gavazzi et al. the gas is driven into the Galactic centre and triggers a starburst, it is difficult to imagine here that the same would occur in our case because the quantities of gas involved are so much larger and its conversion into stars would leave remarkable signatures.

Associating the shutdown in the star formation of the MW with both the end of the thick-disk building phase and a bar is a very attractive solution (e.g. Masters et al. 2012; Cheung et al. 2013). However, as we noted, the bar cannot do this 
by redistributing the gas permanently because the star formation needs to be re-ignited after $\sim 1-2$ Gyr. Instead, we propose that the bar simply inhibits star formation by shearing the gaseous disk, which through viscosity is transferred into turbulence (Fleck 1981; Renaud et al. 2015). The high level of turbulence then provides pressure support for the gas in the disk, preventing it from being swept into the circum-nuclear region of the MW (Athanassoula et al. 2013).

To investigate this possibility, we adopted a simple twophase disk model. One disk is purely stellar with an exponential scale length of $1.8 \mathrm{kpc}$, the other is purely gas with a scale length of $3.8 \mathrm{kpc}$ (Bovy et al. 2012b). Both disks are assumed to have equal masses, a total mass of $5 \times 10^{10} M_{\odot}$, which is approximately the current stellar mass of the MW, the rotation speeds of the disks are $170 \mathrm{~km} \mathrm{~s}^{-1}$ and $220 \mathrm{~km} \mathrm{~s}^{-1}$, and represent the thick and thin disks, respectively. If both disks have constant stellar velocity dispersion, we find from investigating the twofluid stability of such a disk (see Elmegreen 1995) that the disk is Toomre stable, that is, it has $Q \sim 1$ for a gas dispersion of $\sim 50 \mathrm{~km} \mathrm{~s}^{-1}$ or higher everywhere in the disk. The disk is most unstable to two-fluid instabilities at a radius of about $3 \mathrm{kpc}$, and this region requires the highest energy input to stabilize the disk. Changing the assumptions in this model within the observational constraints available, such as increasing the disk scale-lengths (for instance, to 2 and $5 \mathrm{kpc}$ for the stellar and gas disk, respectively), little changes the exact value of the velocity dispersion needed to stabilize the gas disk (i.e. $Q \gtrsim 1$ ).

The flat rotation curve of the gas disk with a velocity of $220 \mathrm{~km} \mathrm{~s}^{-1}$ at $3 \mathrm{kpc}$ has a local shear rate of about $80 \mathrm{~km} \mathrm{~s}^{-1} \mathrm{kpc}^{-1}$. If we assume that the bar has a co-rotation radius of about $5 \mathrm{kpc}$ (see Gerhard 2011, for a discussion about the uncertainty in the co-rotation radius, with reported values between 3.4 and $7 \mathrm{kpc}$; Wegg et al. 2015, found that co-rotation must be beyond $5 \pm 0.2 \mathrm{kpc}$.), then the shear rate at $3 \mathrm{kpc}$ is $\approx \Delta V / \Delta r$, where $\Delta V$ is the velocity difference between the bar and gaseous disk, which is approximately $100 \mathrm{~km} \mathrm{~s}^{-1} \mathrm{kpc}^{-1}$. The shear generated by the bar is stronger than or equal to that within the gaseous disk itself. If we assumed a larger co-rotation radius, the contribution of the bar to the shearing rate would be proportionally higher (it increases linearly with radius). If we assume that the gaseous disk has a thickness of about $300 \mathrm{pc}$ (which is approximately the scale height of the old stars in the thin disk today), we can estimate the velocity dispersion of the clouds by equating the energy of the shear to that dissipated by turbulence as the shear rate times the disk thickness (see Fleck 1981), yielding $180 \mathrm{~km} \mathrm{~s}^{-1} \mathrm{kpc}^{-1} * 0.3 \mathrm{kpc} \approx 50 \mathrm{~km} \mathrm{~s}^{-1}$. Of course, a key parameter to determine whether this idea is energetically feasible is the dissipation time of the turbulence generated by the shear from the gas disk rotation and the bar. The cloud-cloud collision time is $\approx 50-100 \mathrm{Myr}$ (the cloud-cloud dispersion within the ensemble of clouds will decay on several cloud-cloud collision timescales; e.g. Silk \& Norman 2009). The rotation time of the bar is about $180 \mathrm{Myr}$ (compatible with the wide range of the measured values of the present-day bar pattern speed, Gerhard 2011), while the stirring from the shear in the flat rotation curve of the gas disk is injected constantly. Given the similarity of the timescales and the long cloud-cloud collision time, it is plausible that the bar plus disk can generate sufficient shear over approximately the necessary timescales to maintain the turbulence that makes the disk globally stable. To sustain the high rate of shear for the duration of the pause (taking into account the decreasing phase of the SFR, this is from about 10 to $7 \mathrm{Gyr}$ ) requires the bar to exist for about 15 rotation periods, which is plausible.
Of course, our arguments are qualitative and our discussion was only intended to investigate the plausibility of such a scenario. The scenario has some parameters in it for which we used the best observational constraints available (in the rotation speeds, scale lengths, disk thicknesses, velocity dispersions, etc.). We are sensitive to a few of them. For example, if the disk is thinner than we have assumed, $<0.3 \mathrm{kpc}$, the turbulence velocities will be lower, as would the shear be if we assumed a shorter bar. However, highly turbulent velocities would naturally thicken the gas disk, and $0.3 \mathrm{kpc}$ is approximately that of the old thin-disk stars (Bovy et al. 2012b). The co-rotation radius of the bar must be larger than $3 \mathrm{kpc}$ or it will not enhance the turbulence where $Q$ reaches its minimum, and it must be sufficiently long to inhibit star formation out to large radii, although the gas disk is stable out to large radii with only a modest enhancement in the turbulent velocity $\left(20 \mathrm{~km} \mathrm{~s}^{-1}\right)$, and so this is a weak constraint. While in detail we are sensitive to the exact numbers we have chosen, they are plausible given the observations and are not such that they must have particular values for our scenario to be plausible.

We envision a transfer of energy from the large-scale shear into the random cloud motions and then into turbulence in the clouds themselves. While a detailed discussion of this is beyond the scope of this paper, the transfer of energy may prevent the gaseous disk from being gravitationally unstable on any scale (from the disk down to individual clouds, Guillard et al. 2015). If the bar weakens over time, then its effectiveness of shearing the gas will diminish, allowing the thin disk to begin forming stars. As noted in Haywood et al. (2013), it may be that the thin disk started forming in its outer regions, which would be a natural outcome of using the bar to stir the inner-disk gas, maintaining its stability. We also note that a bar in the thick disk will not be similar to the bars observed or modelled locally. Bars in local disks are composed of thin-disk stars and co-rotate with both the gas and stars at this (co-rotation) radius. At co-rotation, gas piles up as a result of orbital crowding at the end of the bar, the piledup gas fragments, eventually leading to star formation (Renaud et al. 2015). In the scenario proposed here, the bar still has a orbital velocity even at its ends that is much lower than the gas, which will then not lead to orbital crowding, but will generate significant shear. In addition, again, unlike simulations of bars consistent with local galaxies, the turbulence generated by a bar as proposed here is significant compared to the rotational support of the gas disk (50 $\mathrm{km} \mathrm{s}^{-1}$ compared to $10-20 \mathrm{~km} \mathrm{~s}^{-1}$; Renaud et al. 2015). Thus, instead of the gas falling deeper into the potential well after the development of a bar, the turbulent pressure partially compensates for the loss of rotational support in a highdispersion system as proposed here (e.g. Burkert et al. 2010). This overcomes our previous objection that the bar will sweep gas into the centre when quenching the galaxy (Athanassoula et al. 2013; Gavazzi et al. 2015), which would be inconsistent with the chemical continuity between the stars of the young thick and old thin disks. In our scenario, star formation is prevented by turbulent support, which similarly supports the gas from generally being swept into the Galactic centre through loss of rotational support and dissipation.

\subsection{Mass-quenching in MW-type galaxies is thick-disk building}

van Dokkum et al. (2013) and Morishita et al. (2015) found consistently that MW-type galaxies build their disks in a self-similar way at $z>1$. This is what we would expect if these objects were building their thick disks, growing in mass while keeping their 
scale length at a constant value. For the MW, it has been argued in Haywood et al. $(2013,2015)$ that the thick disk grew in mass while keeping its scale length constant at $\sim 1.8 \mathrm{kpc}$ from an age greater than 12 Gyr to $\sim 9$ Gyr (or $z>2$ to $z=1.6$ ). That is, the MW disk did not form inside-out, but in a self-similar way, as found by van Dokkum et al. (2013) and Morishita et al. (2015) for MW progenitors. These results strongly indicate that the first substantial component formed in these objects were thick disks, and therefore that quenching is related to thick-disk building and not to the build-up of classical bulges in MW-type galaxies. This is also expected from statistics of bulges and thick disks in the local Universe because of the ubiquity of thick disks in galaxies (Comerón et al. 2011) and the rarity of true classical bulges in local galaxies: the local volume $(<11 \mathrm{Mpc})$ is dominated by pure disk galaxies (Kormendy et al. 2010; Fisher \& Drory 2010; see also Laurikainen et al. 2014). Recent results, shown in Di Matteo et al. $(2014,2015)$, for example, have confirmed the view that the MW is a pure disk galaxy, adding consistency to the overall picture. This statement is therefore likely to be valid more generally for late-type galaxies, first because classical bulges are rare among them, but also because thick disks are observed to be relatively more massive in lower-mass disk galaxies (Comerón et al. 2011).

In contrast, more massive galaxies seem to grow inside-out (van Dokkum et al. 2010; Morishita et al. 2015), with massive bulges already in place at $z \sim 2.4$ (Morishita et al. 2015). This is what we would expect if these objects first formed a bulge, then built their disk. We note that for disks galaxies with stellar masses higher than that of the MW, classical bulges are a more substantial fraction of their total stellar masses. S0-Sb galaxies, which tend to be more massive, usually have high bulge-to-totalmass ratios. These massive bulges and high bulge-to-disk-mass ratios are difficult to explain through secular growth processes alone (see Kormendy \& Kennicutt 2004). These galaxies mainly contain a classical bulge in their central regions and are most likely the descendants of at least a fraction of the massive galaxies that are observed by van Dokkum et al. (2010) and Morishita et al. (2015). This has to be the case because they generally selected their galaxy sample through abundance matching (selected to have densities lower than a constant co-moving density threshold independent of redshift), and those samples contain galaxies that will no doubt be substantially more massive than the MW at $z=0$.

If the phenomenon of quenching is related to the build-up of the central parts of galaxies, it is therefore tempting to suggest that thick disks may be more generally related to quenching for galaxies on the low-mass side $\left(\lessgtr M^{*}\right)$ of the stellar density distribution of galaxies. In contrast, spirals similar to M31 or more massive usually contain a significant classical bulge $(\mathrm{B} / \mathrm{T}=0.31$ for M31, see Kormendy et al. 2010), but have relatively low comoving space densities. Quenching could be linked to classical bulges in larger spirals and more generally in massive galaxies with large $\mathrm{B} / \mathrm{T}$, or galaxies on the high-mass side of the galaxy stellar mass distribution $\left(>M^{*}\right)$.

\section{Conclusions}

Because $[\alpha / \mathrm{Fe}]$ abundances are tightly correlated with age, their number distribution is an accurate record of the past star formation intensity in our Galaxy. Snaith et al. (2014, 2015) used the fact that the age- $[\alpha / \mathrm{Fe}]$ relation contains a fossil record of the past star formation intensity to derive a SFH of the MW inner disk from a local sample of stars. In the present study, we showed that the extensive abundance data from the recent
APOGEE survey confirm our previous study and in particular provide strong evidence of a lull in the SFH, similar to the phenomenon of quenching of high-redshift galaxies.

Quenching is a phenomenon that may be a generic phase in the life of galaxies, and we found that the MW offers the best opportunity to study quenching in significant detail. To our knowledge, this is the first time that a specific episode in the general evolutionary history of galaxies is this directly confirmed from the archaeological record embedded in the stars of our own Galaxy. $\alpha$-elemental abundances show that a significant drop, by a factor of 10 , and long pause in the star formation activity occurred in the MW between the age of $9.5 \mathrm{Gyr}(z \sim 1.6)$ and 7 Gyr $(z=0.8)$, with an almost complete quenching at $z \sim 1.0$. This event manifests itself in the lower density of stars along the inner-disk $\alpha$-elemental sequence between $\sim 0.07$ and 0.17 dex. This dip in the $[\alpha / \mathrm{Fe}]$ abundance distribution, which occurs along the inner-disk sequence, separating the thick disk and the inner thin disk, has been attributed to the combined effect of the selection function and volume sampling of the stars. We argued here that this dip is a real astrophysical phenomenon and that the corresponding lull in the SFH is detected in several independent studies.

This quenching episode marks the end of the thick-disk formation and is followed by a more quiescent phase of star formation in our Galaxy. Although this event marks a clear discontinuity between the formation of the thick and thin disks, we emphasize that there is elemental abundance continuity between the two populations, so that the end of one phase is related to the beginning of the next despite the long pause in the MW's star formation. Hence, the origin of quenching does not appear to be related to a shortage of gas in our Galaxy, otherwise the necessary replenishment of the gas to re-ignite star formation would have left a discontinuity between the abundance properties of the thick and thin disks that we do not observe.

Although we were unable to associate this quenching episode with any of the processes that have been proposed for the quenching in disks, we emphasize that quenching in the MW is not the gradual transition to quiescence, but seems to operate in a similar way and over a similar timescale as in early-type galaxies. We proposed a scenario that might link the cessation of star formation to the development of a strong bar in the thick disk, and its subsequent weakening allowed star formation to re-ignite.

On the basis of the close similarity between the evolution of the MW and MW progenitors and from the demographics of thick disk and classical bulges in the local Universe, we argue that the quenching of the star formation activity for late-type galaxies is associated with the cessation of the growth of thick disks and perhaps with the start of the growth of thin disks.

Acknowledgements. We are grateful to the referee for a detailed report and helpful comments. M.D.L. would like to thank Pierre Guillard for interesting and very helpful discussions on shear and turbulence in galaxy disks.

\section{References}

Abramson, L. E., Kelson, D. D., Dressler, A., et al. 2014, ApJ, 785, L36 Adibekyan, V. Z., Sousa, S. G., Santos, N. C., et al. 2012, A\&A, 545, A32 Aravena, M., Carilli, C., Daddi, E., et al. 2010, ApJ, 718, 177

Athanassoula, E., Machado, R. E. G., \& Rodionov, S. A. 2013, MNRAS, 429, 1949

Baldry, I. K., \& Glazebrook, K. 2003, ApJ, 593, 258

Bell, E. F., van der Wel, A., Papovich, C., et al. 2012, ApJ, 753, 167

Bensby, T., Alves-Brito, A., Oey, M. S., Young, D., \& Melendez, J. 2011, ApJ, 735, L46

Bensby, T., Feltzing, S., \& Oey, M. S. 2014, A\&A, 562, A71 
Bernkopf, J., \& Fuhrmann, K. 2006, MNRAS, 369, 673

Béthermin, M., Daddi, E., Magdis, G., et al. 2015, A\&A, 573, A113 Birnboim, Y., \& Dekel, A. 2003, MNRAS, 345, 349

Blanton, M. R. 2006, ApJ, 648, 268

Bluck, A. F. L., Mendel, J. T., Ellison, S. L., et al. 2014, MNRAS, 441, 599

Bovy, J., Rix, H.-W., \& Hogg, D. W. 2012a, ApJ, 751, 131

Bovy, J., Rix, H.-W., Liu, C., et al. 2012b, ApJ, 753, 148

Bovy, J., Rix, H.-W., Hogg, D. W., et al. 2012c, ApJ, 755, 115

Bovy, J., Nidever, D. L., Rix, H.-W., et al. 2014, ApJ, 790, 127

Bovy, J., Rix, H.-W., Schlafly, E. F., et al. 2015, ApJ, submitted [arXiv: 1509.05796]

Burkert, A., Truran, J. W., \& Hensler, G. 1992, ApJ, 391, 651

Cheng, J. Y., Rockosi, C. M., Morrison, H. L., et al. 2012, ApJ, 752, 51

Cheung, E., Athanassoula, E., Masters, K. L., et al. 2013, ApJ, 779, 162

Chiappini, C., Matteucci, F., \& Gratton, R. 1997, ApJ, 477, 765

Chiappini, C., Matteucci, F., Beers, T. C., \& Nomoto, K. 1999, ApJ, 515, 226

Cignoni, M., Degl'Innocenti, S., Prada Moroni, P. G., \& Shore, S. N. 2006, A\&A, 459, 783

Cimatti, A., Daddi, E., Renzini, A., et al. 2004, Nature, 430, 184

Cimatti, A., Daddi, E., \& Renzini, A. 2006, A\&A, 453, L29

Comerón, S., Elmegreen, B. G., Knapen, J. H., et al. 2011, ApJ, 741, 28

Conroy, C., van Dokkum, P. G., \& Kravtsov, A. 2015, ApJ, 803, 77

Croton, D. J., Springel, V., White, S. D. M., et al. 2006, MNRAS, 367, 864

Daddi, E., Bournaud, F., Walter, F., et al. 2010, ApJ, 713, 686

Dannerbauer, H., Daddi, E., Riechers, D. A., et al. 2009, ApJ, 698, L178

Dehnen, W. 2000, AJ, 119, 800

Di Matteo, P., Haywood, M., Gómez, A., et al. 2014, A\&A, 567, A122

Di Matteo, P., Gómez, A., Haywood, M., et al. 2015, A\&A, 577, A1

Elmegreen, B. G. 1995, MNRAS, 275, 944

Fisher, D. B., \& Drory, N. 2010, ApJ, 716, 942

Fleck, R. C., Jr. 1981, ApJ, 246, L151

Fontaine, G., Brassard, P., \& Bergeron, P. 2001, PASP, 113, 409

Förster Schreiber, N. M., Genzel, R., Newman, S. F., et al. 2014, ApJ, 787, 38

Fuhrmann, K. 2004, Astron. Nachr., 325, 3

Fuhrmann, K. 2011, MNRAS, 414, 2893

Gabor, J. M., \& Bournaud, F. 2014, MNRAS, 441, 1615

Gabor, J. M., Davé, R., Finlator, K., \& Oppenheimer, B. D. 2010, MNRAS, 407, 749

Gavazzi, G., Consolandi, G., Dotti, M., et al. 2015, A\&A, 580, A116

Gerhard, O. 2011, Mem. Soc. Astron. It. Supp., 18, 185

Gilmore, G., \& Wyse, R. F. G. 1986, Nature, 322, 806

Gilmore, G., \& Wyse, R. F. G. 1991, ApJ, 367, L55

Granato, G. L., De Zotti, G., Silva, L., Bressan, A., \& Danese, L. 2004, ApJ, 600,580

Gratton, R., Carretta, E., Matteucci, F., \& Sneden, C. 1996, Formation of the Galactic Halo... Inside and Out, 92, 307

Gratton, R. G., Bragaglia, A., Carretta, E., et al. 2003, A\&A, 408, 529

Guillard, P., Boulanger, F., Lehnert, M. D., et al. 2015, A\&A, 574, A32

Hallé, A., Di Matteo, P., Haywood, M., \& Combes, F. 2015, A\&A, 578, A58

Hansen, B. M. S., Anderson, J., Brewer, J., et al. 2007, ApJ, 671, 380

Hayden, M. R., Holtzman, J. A., Bovy, J., et al. 2014, AJ, 147, 116

Hayden, M. R., Bovy, J., Holtzman, J. A., et al. 2015, ApJ, 808, 132

Haywood, M. 2001, MNRAS, 325, 1365

Haywood, M. 2008, MNRAS, 388, 1175

Haywood, M. 2014a, Mem. Soc. Astron. It. Supp., 85, 253

Haywood, M. 2014b, To appear in Lessons from the local group - A Conference in Honour of David Block and Bruce Elmegreen, eds. K. C. Freeman, B. G. Elmegreen, D. L. Block, \& M. Woolway (New York: Springer)

Haywood, M., Di Matteo, P., Lehnert, M. D., Katz, D., \& Gómez, A. 2013, A\&A, 560, A109
Haywood, M., Di Matteo, P., Snaith, O., \& Lehnert, M. D. 2015, A\&A, 579, A5 Holtzman, J. A., Shetrone, M., Johnson, J. A., et al. 2015, AJ, 150, 148 Kajisawa, M., Morishita, T., Taniguchi, Y., et al. 2015, ApJ, 801, 134 Kalirai, J. S. 2012, Nature, 486, 90

Kaviraj, S., Kirkby, L. A., Silk, J., \& Sarzi, M. 2007, MNRAS, 382, 960 Kilic, M., Munn, J. A., Williams, K. A., et al. 2010, ApJ, 715, L21 Kobayashi, C., Karakas, A. I., \& Umeda, H. 2011, MNRAS, 414, 3231 Kordopatis, G., Wyse, R. F. G., Gilmore, G., et al. 2015, A\&A, 582, A122 Kormendy, J., \& Kennicutt, R. C., Jr. 2004, ARA\&A, 42, 603 Kormendy, J., Drory, N., Bender, R., \& Cornell, M. E. 2010, ApJ, 723, 54 Kroupa, P. 2001, MNRAS, 322, 231

Kunder, A., Koch, A., Rich, R. M., et al. 2012, AJ, 143, 57

Lang, P., Wuyts, S., Somerville, R. S., et al. 2014, ApJ, 788, 11

Laurikainen, E., Salo, H., Athanassoula, E., Bosma, A., \& Herrera-Endoqui, M. 2014, MNRAS, 444, L80

Lehnert, M. D., Di Matteo, P., Haywood, M., \& Snaith, O. N. 2014, ApJ, 789, L30

Licquia, T. C., Newman, J. A., \& Brinchmann, J. 2015, ApJ, 809, 96

Majewski, S. R., Schiavon, R. P., Frinchaboy, P. M., et al. 2015, AJ, submitted [arXiv: 1509.05420$]$

Mancini, C., Renzini, A., Daddi, E., et al. 2015, MNRAS, 450, 763

Martig, M., Bournaud, F., Teyssier, R., \& Dekel, A. 2009, ApJ, 707, 250

Martin, D. C., Wyder, T. K., Schiminovich, D., et al. 2007, ApJS, 173, 342

Masters, K. L., Nichol, R. C., Haynes, M. P., et al. 2012, MNRAS, 424, 2180

Matteucci, F., \& Francois, P. 1989, MNRAS, 239, 885

Melvin, T., Masters, K., Lintott, C., et al. 2014, MNRAS, 438, 2882

Minchev, I., Chiappini, C., \& Martig, M. 2014, A\&A, 572, A92

Morishita, T., Ichikawa, T., Noguchi, M., et al. 2015, ApJ, 805, 34

Mutch, S. J., Croton, D. J., \& Poole, G. B. 2011, ApJ, 736, 84

Nissen, P. E. 2015, A\&A, 579, A52

Nissen, P. E., \& Schuster, W. J. 2010, A\&A, 511, L10

Nelson, E. J., van Dokkum, P. G., Momcheva, I., et al. 2013, ApJ, 763, L16

Nidever, D. L., Bovy, J., Bird, J. C., et al. 2014, ApJ, 796, 38

Papovich, C., Labbé, I., Quadri, R., et al. 2015, ApJ, 803, 26

Popping, G., Caputi, K. I., Trager, S. C., et al. 2015, MNRAS, 454, 2258

Recio-Blanco, A., de Laverny, P., Kordopatis, G., et al. 2014, A\&A, 567, A5

Reddy, B. E., Lambert, D. L., \& Allende Prieto, C. 2006, MNRAS, 367, 1329

Reid, I. N. 2005, ARA\&A, 43, 247

Renaud, F., Bournaud, F., Emsellem, E., et al. 2015, MNRAS, 454, 3299

Rowell, N. 2013, MNRAS, 434, 1549

Santiago, B. X., Brauer, D. E., Anders, F., et al. 2016, MNRAS, 585, A42

Schawinski, K., Urry, C. M., Simmons, B. D., et al. 2014, MNRAS, 440, 889

Shen, J., Rich, R. M., Kormendy, J., et al. 2010, ApJ, 720, L72

Schönrich, R., \& Binney, J. 2009, MNRAS, 399, 1145

Schultheis, M., Zasowski, G., Allende Prieto, C., et al. 2014, AJ, 148, 24

Sheth, K., Elmegreen, D. M., Elmegreen, B. G., et al. 2008, ApJ, 675, 1141

Sil'chenko, O. 2013, Mem. Soc. Astron. It. Supp., 25, 93

Silk, J., \& Norman, C. 2009, ApJ, 700, 262

Snaith, O. N., Haywood, M., Di Matteo, P., et al. 2014, ApJ, 781, L31

Snaith, O., Haywood, M., Di Matteo, P., et al. 2015, A\&A, 578, A87

Tacconi, L. J., Genzel, R., Neri, R., et al. 2010, Nature, 463, 781

Tacconi, L. J., Neri, R., Genzel, R., et al. 2013, ApJ, 768, 74

Timmes, F. X., Woosley, S. E., \& Weaver, T. A. 1995, ApJS, 98, 617

Torres, S., García-Berro, E., Althaus, L. G., \& Camisassa, M. E. 2015, A\&A, 581, A90

VandenBerg, D. A., Bond, H. E., Nelan, E. P., et al. 2014, ApJ, 792, 110

van Dokkum, P. G., Whitaker, K. E., Brammer, G., et al. 2010, ApJ, 709, 1018

van Dokkum, P. G., Leja, J., Nelson, E. J., et al. 2013, ApJ, 771, L35

Wegg, C., Gerhard, O., \& Portail, M. 2015, MNRAS, 450, 4050

Whitaker, K. E., Franx, M., Bezanson, R., et al. 2015, ApJ, 811, L12 